\title{
V1 orientation plasticity is explained by broadly tuned feedforward inputs and intracortical sharpening
}

\author{
ANDREW F. TEICH,,$^{1,2}$ AND NING QIAN ${ }^{2,3}$ \\ ${ }^{1}$ Department of Pathology, Columbia University, New York, New York \\ ${ }^{2}$ Department of Neuroscience, Columbia University, New York, New York \\ ${ }^{3}$ Department of Physiology and Cellular Biophysics, Columbia University, New York, New York
}

(RECEIVED October 18, 2009; ACCEPTED February 11, 2010)

\begin{abstract}
Orientation adaptation and perceptual learning change orientation tuning curves of V1 cells. Adaptation shifts tuning curve peaks away from the adapted orientation, reduces tuning curve slopes near the adapted orientation, and increases the responses on the far flank of tuning curves. Learning an orientation discrimination task increases tuning curve slopes near the trained orientation. These changes have been explained previously in a recurrent model (RM) of orientation selectivity. However, the RM generates only complex cells when they are well tuned, so that there is currently no model of orientation plasticity for simple cells. In addition, some feedforward models, such as the modified feedforward model (MFM), also contain recurrent cortical excitation, and it is unknown whether they can explain plasticity. Here, we compare plasticity in the MFM, which simulates simple cells, and a recent modification of the RM (MRM), which displays a continuum of simple-to-complex characteristics. Both pre- and postsynaptic-based modifications of the recurrent and feedforward connections in the models are investigated. The MRM can account for all the learning- and adaptation-induced plasticity, for both simple and complex cells, while the MFM cannot. The key features from the MRM required for explaining plasticity are broadly tuned feedforward inputs and sharpening by a Mexican hat intracortical interaction profile. The mere presence of recurrent cortical interactions in feedforward models like the MFM is insufficient; such models have more rigid tuning curves. We predict that the plastic properties must be absent for cells whose orientation tuning arises from a feedforward mechanism.
\end{abstract}

Keywords: Adaptation, Learning, Presynaptic, Postsynaptic, Tuning curve

\section{Introduction}

Strong orientation selectivity first emerges in V1 (Hubel \& Wiesel, 1962). V1 orientation tuning is not static but can be modified by adaptation and perceptual learning. Specifically, for cells whose preferred orientations are near the adapted orientation, adaptation generates (1) a peak shift away from the adapted orientation, (2) shallower slopes around the adapted orientation, and (3) increased responses on the far side of the tuning curves that face away from the adapted orientation (Dragoi et al., 2000, 2001; Felsen et al., 2002) (but see Muller et al., 1999, and Kohn \& Movshon, 2004). For cells whose preferred orientations are far away from the adapted orientation, there is (4) a modest sharpening of their tuning curves. Perceptual learning of an orientation discrimination task also changes orientation tuning curves but differently. Schoups et al. (2001) reported that for cells near the trained orientation, there is (5) a sharpening of the tuning curves around the trained orientation, and for cells further away from the trained orientation, there is (6) a modest broadening of the tuning curves (but see Ghose et al., 2002, and discussions in Teich \& Qian,

Address correspondence and reprint requests to: Dr. Andrew F. Teich, Division of Neuropathology, Department of Pathology, Columbia University, 630 West 168th Street, PH 15 Stem-Room 124, New York, NY 10032. E-mail: aft25@columbia.edu 2003a). Similar learning-induced changes have been found in V4 (Yang \& Maunsell, 2004; Raiguel et al., 2006).

We previously explained all the above six tuning curve changes with a recurrent model (RM) of orientation selectivity (Teich \& Qian, 2003a). The RM assumes that feedforward input to a V1 cell is broadly tuned for orientation and is then sharpened by recurrent intracortical excitation and inhibition (Ben-Yishai et al., 1995; Douglas et al., 1995; Somers et al., 1995; Carandini \& Ringach, 1997). We simulated the adaptation- and the learning-induced changes by depressing the connections in the RM in different ways (Teich \& Qian, 2003a). In fact, we predicted the learning-induced changes of tuning curves (Qian \& Matthews, 1999) before the recorded data (Schoups et al., 1998) were reanalyzed in light of our model to reveal the changes (Schoups et al., 2001).

We showed subsequently that the model cells in the RM are complex when their orientation tuning is as sharp as typical V1 cells (Teich \& Qian, 2006). Thus, our previous plasticity model is for complex cells, and there is currently no model of orientation plasticity for simple cells. In their physiological study of orientation plasticity, Dragoi et al. (2000) recorded from a large number of cat V1 cells, but they did not classify the cells into simple or complex. However, they noted that they recorded cells from all layers of V1 and did not find a layer dependence of plasticity. Since the V1 input layer is dominated by simple cells, it is likely that many of their 
recorded cells are simple, and these simple cells showed the same orientation plasticity as complex cells (but see Muller et al.'s, 1999, study of monkey V1). There is thus a need to explain orientation plasticity in a simple cell model. This is particularly important when the origin of orientation selectivity of the input-layer simple cells is concerned (see Discussion).

Moreover, it is unclear whether the other major class of orientation models, namely the feedforward models, can also explain all the plastic properties listed above or not. Unlike the RM, the feedforward models posit that V1 orientation tuning arises exclusively from the alignment of feedforward inputs. For a strictly feedforward model without intracortical interactions (Hubel \& Wiesel, 1962), a cell's preferred orientation is anatomically determined by the alignment axis of feedforward inputs. Therefore, the model cannot explain, for example, the postadaptation peak shift unless adaptation is assumed to alter the axis. However, Troyer et al. (1998) proposed a modified feedforward model (MFM) in which they introduced cortical antiphase inhibition to achieve contrast invariance and recurrent cortical excitation to boost responses. Although it is generally recognized that recurrent cortical connections are necessary to explain plasticity (Dragoi et al., 2000; Felsen et al., 2002; Teich \& Qian, 2003a), it is not clear whether their presence in the MFM is sufficient for this feedforward model to show plasticity too. Felsen et al. (2002) previously examined plasticity of the MFM, but they did not implement the recurrent excitation in the original MFM. In addition, they only considered one of the six plastic properties (adaptation-induced peak shift) mentioned above.

Since the MFM shows simple cell characteristics (Troyer et al., 1998; Teich \& Qian, 2006), in this study, we examine whether it provides a viable plasticity model for simple cells. For comparison, we also investigate plastic properties of the modified recurrent model (MRM), a new model we proposed recently that extends the RM to display a continuum of simple-to-complex characteristics (Teich \& Qian, 2006). In our previous study of plasticity in the RM (Teich \& Qian, 2003a), we only considered modifying connection strengths based on the activity of postsynaptic cells. Here, we consider both the pre- and the postsynaptic rules of modification. We demonstrate that orientation plasticity provides a powerful tool for differentiating feedforward and recurrent mechanisms of orientation selectivity. Finally, we provide an intuitive understanding of what features of a model are essential to account for the observed plasticity. As new models for orientation tuning are still being proposed, such an intuitive understanding will provide guidance on whether a new model is consistent with the plasticity data. Preliminary results have been published in abstract form (Teich \& Qian, 2007).

\section{Materials and methods}

The MFM and MRM are identical to those described in Teich and Qian (2006). Both models start with a field of lateral geniculate nucleus (LGN) cells, and the LGN response to a stimulus is then fed to a cortical network of V1 cells. The LGN cells are the same in each model; the main difference between the models is found in the aspect ratio of the thalamocortical connections and at the connectivity patterns among the V1 cells. We first outline both models. For further details, please see the methods section of Teich and Qian (2006) and Appendix 1. We then describe how the connections in the models were modified to simulate learning and adaptation. All simulations were performed with Matlab (Mathworks, Natick, MA) on either a Linux or a PC computer.

\section{Basic features common to both models}

LGN responses to stimuli

The LGN cells in both models closely follow the design of Troyer et al. (1998) but have a simplified temporal kernel, taken from Chen et al. (2001). We consider 240 ON-center and 240 OFFcenter cells arranged in a grid. Each cell's spatial receptive field is center-surround, modeled with a difference of Gaussians:

$$
f(r)=\left(17 / \sigma_{\text {center }}^{2}\right) e^{-r^{2} / \sigma_{\text {center }}^{2}}-\left(16 / \sigma_{\text {surround }}^{2}\right) e^{-r^{2} / \sigma_{\text {surround }}^{2}}
$$

where $r$ is the radial distance from the center of the receptive field, $\sigma_{\text {center }}=15^{\prime}$ and $\sigma_{\text {surround }}=60^{\prime}$.

We use stationary light bars as stimuli. The bars have a width of $30 \mathrm{~min}$ and a length spanning the entire receptive field for all model cells. The LGN responses to a given stimulus are first determined through a spatiotemporal convolution of the stimulus with the cell's spatial receptive field and temporal kernel and then scaled using a contrast response curve (Teich \& Qian, 2006). We assume a stimulus contrast of $50 \%$.

\section{Cortical receptive fields}

The connection from an LGN cell at $(x, y)$ to a vertically oriented V1 cell is determined by a Gabor function of the form:

$$
g(x, y)=\exp \left[-\left(x^{2} /\left(2 \sigma_{x}^{2}\right)\right)-\left(y^{2} /\left(2 \sigma_{y}^{2}\right)\right)\right] \times \cos (\omega x+\varphi),
$$

where $\omega /(2 \pi)=0.8$ cycles/deg is the spatial frequency, $\phi$ determines the $\mathrm{V} 1$ receptive-field phase, and $\sigma_{\mathrm{x}}$ and $\sigma_{\mathrm{y}}$ determine the receptive-field size. For V1 cells with orientation $\theta$, the Gabor connectivity function $g(x, y, \theta)$ is obtained from $g(x, y)$ via proper rotation. The Gabor function specifies both the orientation and the phase of the V1 cell's receptive field, with positive values representing connections from ON-center LGN cells and negative values representing connections from OFF-center LGN cells. Thus, the connection $C(x, y ; \theta, \phi)$ from an ON-center (OFF-center) LGN cell at $(x, y)$ to a $\mathrm{V} 1$ cell indexed at preferred orientation $\theta$ and spatial phase $\phi$ is equal to the positive (negative) part of $g(x, y, \theta)$. The aspect ratio is defined as the ratio of the Gaussian length (the $5 \%$ points of the peak, along the long axes of the Gaussian) to the width of a half-cycle of the sinusoid. A large aspect ratio equals a welltuned LGN input to V1 cells, and a smaller aspect ratio equals a broadly tuned LGN input. For the MFM, we use the same aspect ratio (4.54) as Troyer et al. (1998). For the MRM, we use an aspect ratio of 2, which was used by Somers et al. (1995).

\section{Intracortical circuitry}

Both models have V1 excitatory cells and inhibitory cells at eight evenly spaced spatial phases over $2 \pi$ and 64 evenly distributed orientations over $180 \mathrm{deg}$. The V1 cells are assumed to represent the same area of visual space and draw feedforward input from the same field of LGN cells. Each V1 cell also receives cortical input from other V1 cells. For connections to a given V1 cell, we first normalize all connections from LGN cells to one, all connections from excitatory V1 cells to one, and all connections from inhibitory $\mathrm{V} 1$ cells to one, and then apply different weighting factors to different sets of connections to manipulate their strengths. For example, the connections from excitatory to excitatory cells are weighted by the factor $w_{\mathrm{e} \rightarrow \mathrm{e}}$. The MFM and MRM differ in how cortical cells are connected, and the complete list of weighting 
factors between the different cell types is explained below and in Appendix 1. The specific value of these weights matters less for model performance than their relative ratios; in all the models we present, the results are stable over a wide range of values.

The membrane potential of each cortical cell as a function of time is calculated until a steady-state value is reached. The firing rate is directly related to the membrane potential by applying a threshold and then a gain factor (Carandini \& Ringach, 1997; Teich \& Qian, 2003b, 2006). In each model, we set our parameters so that the LGN input contributes one half to one third of a V1 cell's firing response (Ferster \& Miller, 2000), and V1 excitatory cells have a maximal firing rate somewhere between 30 and $50 \mathrm{~Hz}$ (inhibitory cells fire as high as $100 \mathrm{~Hz}$ in some simulations).

\section{Intracortical circuitry for the MFM}

Following Troyer et al. (1998), we determine the connection between any two cortical cells $a$ [indexed at $\left.\left(\theta^{\prime}, \phi^{\prime}\right)\right]$ and $b$ [indexed at $(\theta, \phi)]$ by computing the normalized correlation between their receptive fields:

$$
c(a ; b)=c^{\prime}(a ; b) / \sqrt{c^{\prime}(a ; a) c^{\prime}(b ; b)},
$$

where $c^{\prime}(a ; b)$ is the raw correlation computed by multiplying the two receptive fields of cells $a$ and $b$ point by point and then summing across all points. Two cortical cells with the exact same orientation and spatial phase have a normalized correlation of 1 and thus have the strongest positive (excitatory) connection; two cortical cells with the exact same orientation and opposite spatial phase (antiphase) have a normalized correlation of -1 and have the strongest negative (inhibitory) connection. All other combinations are intermediate, with the nature of the connection (excitatory or inhibitory) being determined by the sign of the normalized correlation. The final strength of the connection is determined by raising the power of the correlation to a power Npow. Mathematically, the connection strength $C(a ; b)$ from cell $a$ to cell $b$ is based on the normalized correlation according to:

$$
C(a ; b)=[\operatorname{sgn}(a) c(a ; b)]_{+}^{\text {Npow }}
$$

where $\operatorname{sgn}(a)=1$ if $a$ is excitatory and -1 if $a$ is inhibitory, and $[x]_{+}$ $=x$ if $x>0$ and 0 otherwise. Consequently, positive and negative $c(a$; $b$ ) values set excitatory and inhibitory connection strengths, respectively. Npow determines the strength of connectivity as a function of correlation; larger values lead to less connectivity between cells that are weakly correlated. We use an Npow value of 6 for our MFM simulations, which is the default value used by Troyer et al. (1998).

\section{Intracortical circuitry for the MRM}

The MRM was introduced in Teich and Qian (2006). It is essentially a RM with added antiphase inhibition. LGN-to-V1 connectivity with an aspect ratio of 2 is used to deliver broadly tuned feedforward input to cortical excitatory and inhibitory cells that are recurrently connected to one another, just like in the RM (Ben-Yishai et al., 1995; Douglas et al., 1995; Somers et al., 1995; Carandini \& Ringach, 1997; Teich \& Qian, 2003a). Excitatory and inhibitory recurrent connection strengths are assumed to be maximal for cells with similar preferred orientation and decrease with the difference in preferred orientations. However, inhibitory connections are assumed to be somewhat broader than excitatory connections so that if the excitatory and inhibitory profiles are subtracted from each other, cells with similar preferred orientations have a net excitatory influence on each other, whereas cells with a somewhat larger difference in preferred orientation have a net inhibitory influence on each other (the "Mexican hat" connection profile). This causes an enhancement of responses close to a cell's preferred orientation and a suppression of responses far from a cell's preferred orientation, and this is what transforms a broadly tuned feedforward input into a sharply tuned output. For the cells involved in the Mexican hat interaction above, we call the excitatory cells "e" cells and the inhibitory cells "ri" cells (this is to distinguish the recurrent inhibitory cells from antiphase inhibitory cells, which we call "ai" cells). All the connections in the Mexican hat $(\mathrm{e} \rightarrow \mathrm{e}, \mathrm{e} \rightarrow \mathrm{ri}$, $\mathrm{ri} \rightarrow \mathrm{e}$, and $\mathrm{ri} \rightarrow \mathrm{ri}$ ) are based solely on difference in preferred orientation and are spatial phase insensitive. Specifically, the equations that dictate connectivity strength from an "e" or "ri" cell indexed at $\left(\theta^{\prime}, \phi^{\prime}\right)$ to an "e" cell indexed at $(\theta, \phi)$ are

$$
\begin{gathered}
C_{\mathrm{e} \rightarrow \mathrm{e}}\left(\theta^{\prime}, \phi^{\prime} ; \theta, \phi\right)=\exp \left[-\left(\theta-\theta^{\prime}\right)^{2} /\left(2 \sigma_{\mathrm{e}}^{2}\right)\right] \\
C_{\mathrm{ri} \rightarrow \mathrm{e}}\left(\theta^{\prime}, \phi^{\prime} ; \theta, \phi\right)=\exp \left[-\left(\theta-\theta^{\prime}\right)^{2} /\left(2 \sigma_{\mathrm{ri}}^{2}\right)\right],
\end{gathered}
$$

where $\sigma_{\mathrm{e}}=35 \mathrm{deg}$ and $\sigma_{\mathrm{ri}}=52 \mathrm{deg}$. As noted earlier, the connectivity strength from all "e" cells and all "ri" cells to a given cell is each normalized to one before applying specific weighting factors. The same equations can be written for $C_{\mathrm{e} \rightarrow \mathrm{ri}}\left(\theta^{\prime}, \phi^{\prime} ; \theta, \phi\right)$ and $C_{\mathrm{ri} \rightarrow \mathrm{ri}}\left(\theta^{\prime}, \phi^{\prime} ; \theta, \phi\right)$.

We then add a third set of V1 cells that provide antiphase inhibition ("ai" cells). Similarly to the antiphase inhibitory cells in the MFM, the antiphase inhibitory cells in the MRM receive excitatory input from the LGN and same-phase cortical excitation from "e" cells but do not receive any cortical inhibition (either from "ri" or from "ai" cells). These antiphase inhibitory cells then deliver antiphase inhibition to the "e" and "ri" cells using the correlation-based rule from the MFM above [see eqns. (3) and (4)]. The strongest inhibition is to cells of the opposite spatial phase (Troyer et al., 1998). Note that since there are two types of inhibitory cells in the MRM, each type of inhibitory cell connection is separately normalized and weighted. More emphasis on antiphase inhibition causes the model to produce simple cells, whereas more emphasis on recurrent inhibition produces complex cells (see Results for further discussion). For the simple cell MRM, we set the connectivity weights $w_{\mathrm{ai} \rightarrow \mathrm{e}}$ and $w_{\mathrm{ai} \rightarrow \mathrm{ri}}$ to 0.2 and the connectivity weights $w_{\mathrm{ri} \rightarrow \mathrm{e}}$ and $w_{\mathrm{ri} \rightarrow \mathrm{ri}}$ to 3.5 ; for the complex cell MRM, we lower the connectivity weights $w_{\mathrm{ai} \rightarrow \mathrm{e}}$ and $w_{\mathrm{ai} \rightarrow \mathrm{ri}}$ to 0.02 and raise the connectivity weights $w_{\mathrm{ri} \rightarrow \mathrm{e}}$ and $w_{\mathrm{ri} \rightarrow \mathrm{ri}}$ to 3.86 .

\section{Changing connection weights}

Around the trained/adapted orientation, both learning and adaptation reduce a cell's peak response to its preferred orientation, although the cell's responses to other stimulus orientations may increase (see plastic property number 3 in Introduction) (Dragoi et al., 2000; Schoups et al., 2001). We first modify connections in the models to simulate the peak response reduction and then examine the consequent changes to tuning curve shapes. Note that we are not modeling the time course of synaptic modifications. Rather, we are investigating how final patterns of connection changes affect orientation tuning curves, and we are then comparing the results with the experimental data. Throughout the paper, we designate the trained/adapted orientation as $0 \mathrm{deg}$. To reduce peak responses around $0 \mathrm{deg}$, we can either (1) reduce intracortical excitation for 
cells tuned around 0 deg or (2) reduce both intracortical excitation and inhibition for cells tuned around $0 \mathrm{deg}$ while making sure that the net effect is an activity reduction. (A third possibility, considered below, is to reduce feedforward excitation around $0 \mathrm{deg}$.) Note that a pure reduction of inhibition does not work, as that will lead to an increase in peak activity. An increase in inhibition will also cause a decrease in peak activity, and this is another possibility we have tried. We find that computationally, an increase in inhibition is functionally identical to a decrease in excitation, and so we have not included figures for this manipulation in the Results section.

We can reduce connection weights using either a presynaptic rule or a postsynaptic rule (see Fig. 1). A presynaptic rule assumes that all connections from a presynaptic cell are scaled down by a common factor based on the presynaptic cell's recent activity and has its basis in synaptic vesicle depletion (Betz, 1970; Kusano \& Landau, 1975). A postsynaptic rule assumes that all connections to a postsynaptic cell are scaled down by a common factor based on the postsynaptic cell's recent activity and has its basis in the synaptic scaling literature (Abbott \& Nelson, 2000; Turrigiano \& Nelson, 2000; Feldman, 2002); in addition, it has been found that even short periods of adaptation cause a hyperpolarization that could affect all inputs to a postsynaptic cell (Carandini \& Ferster, 1997). Our previous modeling of learning and adaptation has assumed a postsynaptic rule (Teich \& Qian, 2003a). Here, we consider both a preand a postsynaptic rule. Thus, we examine four plasticity conditions: presynaptic reduction of cortical excitation (pre-e), postsynaptic reduction of cortical excitation (post-e), presynaptic reduction of cortical excitation and inhibition (pre-ei), and postsynaptic reduction of cortical excitation and inhibition (post-ei). These four canonical forms of plasticity are by no means mutually exclusive (e.g., one could have pre-e and post-e together), but for simplicity, we keep them separate in this paper.

\section{A. Pre-synaptic}

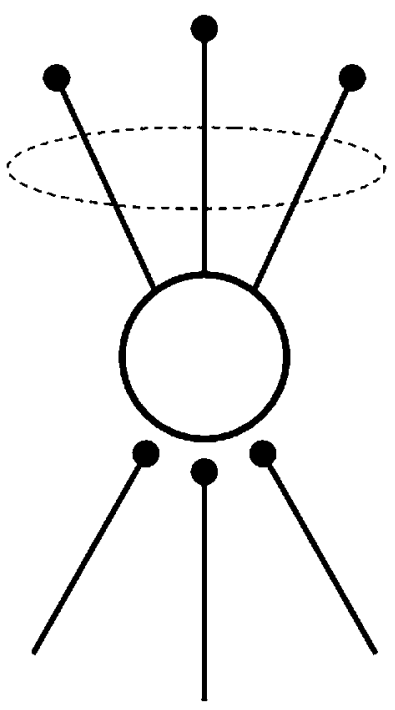

Fig. 1. Presynaptic verses postsynaptic rules. A presynaptic rule (A) assumes that all connections (indicated by the dotted ellipse) from a presynaptic neuron (solid circle) are reduced by a common factor based on the prior activity of this presynaptic neuron. A postsynaptic rule (B) assumes that all connections (indicated by the dotted ellipse) to a postsynaptic neuron (solid circle) are reduced by a common factor based on the prior activity of this postsynaptic cell.
In addition to modifying the intracortical connection weights discussed above, a final possibility for generating an activity reduction around the trained/adapted orientation $(0 \mathrm{deg})$ in V1 is to depress the feedforward connections from the LGN cells to the V1 cells tuned around 0 deg. In this case, a presynaptic rule is inappropriate because the presynaptic LGN cells are not orientation selective. Specifically, a presynaptic scheme predicts that the LGN activities are uniformly depressed by a drifting grating regardless of the orientation of the grating and thus cannot explain activity reduction around a specific orientation in V1. In contrast, a postsynaptic rule for depressing the feedforward connections is feasible because the postsynaptic V1 cells are orientation tuned. We will call this condition post-f. During our simulations for Teich and Qian (2003a), we found that the post-f condition is virtually identical to the post-e condition; in both cases, excitatory input is reduced based on postsynaptic activity. In this sense, we can assume that the post-f condition is included in the post-e category. However, since we previously did not show detailed simulations for the post- $f$ condition, we include this condition here for completeness. More importantly, since orientation tuning in the MFM is determined by feedforward connections, it is interesting to examine how modifications of the feedforward connections affect the tuning curve shapes in the MFM.

Mathematically, the reduction of connection weights is similar to that in our previous plasticity study (Teich \& Qian, 2003a). Plausible patterns of connection changes have to be based on cell's activities during learning/adaptation. A cell's activity, in turn, depends on the difference between its preferred orientation $(\theta)$ and the trained/adapted orientation $(0 \mathrm{deg})$. Since the drifting gratings used in the physiological experiments cover all phases, it is reasonable to assume that the activities are phase independent. Consider the connection from an excitatory cell preferring $\theta^{\prime}$ to an excitatory cell preferring $\theta$. In the pre-e condition, for example, the connection is reduced from the baseline value $w_{\mathrm{e} \rightarrow \mathrm{e}}$ to:

$$
w_{\mathrm{e} \rightarrow \mathrm{e}}\left(\theta^{\prime}, \theta\right)=w_{\mathrm{e} \rightarrow \mathrm{e}} \times\left(1-A_{\mathrm{e}} \exp \left[-\theta^{\prime 2} /\left(2 \sigma_{r}^{2}\right)\right]\right)
$$

according to the presynaptic activity (or $\theta^{\prime}$ ) and independent of the postsynaptic activity (or $\theta$ ). When $\theta^{\prime}=0 \mathrm{deg}$, the connection is reduced maximally by a factor of $A_{\mathrm{e}}$. The reduction gradually trails off with $\theta^{\prime}$ away from $0 \mathrm{deg}$, and the spread is determined by $\sigma_{r}$. A similar expression applies to excitatory connections to an inhibitory cell

$$
w_{\mathrm{e} \rightarrow \mathrm{i}}\left(\theta^{\prime}, \theta\right)=w_{\mathrm{e} \rightarrow \mathrm{i}} \times\left(1-A_{\mathrm{e}} \exp \left[-\theta^{\prime 2} /\left(2 \sigma_{r}^{2}\right)\right]\right),
$$

where the inhibitory cell " $i$ " is antiphase in the MFM but can be either an antiphase or a recurrent inhibitory cell in the MRM. Note that we use the same maximal reduction factor $\left(A_{\mathrm{e}}\right)$ from "e" cells to all other cells in a given simulation.

In contrast, for the post-e condition, the $\theta^{\prime}$ variables on the right hand side of the above equations should all be replaced by $\theta$. To reiterate, the postsynaptic rule reduces all connections to a postsynaptic cell by a common factor depending on $\theta$ but not $\theta^{\prime}$, whereas the presynaptic rule reduces all connections from a presynaptic cell by a common factor depending on $\theta^{\prime}$ but not $\theta$.

For the post-ei and pre-ei conditions, the equations for modifying excitatory connections are the same as those for the post-e and pre-e conditions above. In addition, there are equations for modifying connections from recurrent inhibitory and antiphase inhibitory cells. The equations have exactly the same form as those above but with the maximum reduction factor denoted by $A_{\mathrm{i}}$. Also note that the MRM contains both the (recurrent) inhibition in the Mexican hat 
profile and the antiphase inhibition. For all our simulations with the MRM that involve reducing inhibition, we will only present results that reduce recurrent inhibition in the Mexican hat. We have also done simulations that reduce the antiphase inhibition in the MRM, and we find that this generally does not affect the tuning curve slope or the tuning curve peak location. The reason is discussed in the Results section around Fig. 5. The MFM only has inhibition through antiphase cells, so the pre-ei and post-ei conditions in the MFM necessarily involve reducing antiphase inhibition.

Finally, for the post-f condition, there are a similar set of equations for reducing feedforward connections to excitatory, recurrent inhibitory, and antiphase inhibitory V1 cells:

$$
\begin{gathered}
w_{\mathrm{f} \rightarrow \mathrm{e}}(\theta)=w_{\mathrm{f} \rightarrow \mathrm{e}} \times\left(1-A_{\mathrm{f}} \exp \left[-\theta^{2} /\left(2 \sigma_{r}^{2}\right)\right]\right) \\
w_{\mathrm{f} \rightarrow \mathrm{ri}}(\theta)=w_{\mathrm{f} \rightarrow \mathrm{ri}} \times\left(1-A_{\mathrm{f}} \exp \left[-\theta^{2} /\left(2 \sigma_{r}^{2}\right)\right]\right) \\
w_{\mathrm{f} \rightarrow \mathrm{ai}}(\theta)=w_{\mathrm{f} \rightarrow \mathrm{ai}} \times\left(1-A_{\mathrm{f}} \exp \left[-\theta^{2} /\left(2 \sigma_{r}^{2}\right)\right]\right) .
\end{gathered}
$$

Note that here, only the postsynaptic cells have a preferred orientation $\theta$. As in the simulations for reducing excitation and inhibition, we use the same maximal reduction factor $\left(A_{\mathrm{f}}\right)$ for reducing feedforward input to all cortical cells in a given simulation.

We are able to achieve qualitative agreement with the data over a wide range of $\sigma_{r}$ values. We find that our results are stable for $\sigma_{r}$ values ranging from 10 to 40, with the caveat that at very small $\sigma_{r}$ values (10 or less), some of our models begin to show abnormally pointed tuning curves; this phenomenon has already been discussed in our prior plasticity paper (Teich \& Qian, 2003a). In the following figures, $\sigma_{r}$ was set to 24 for the MRM and 22 for the MFM.

\section{Results}

We compare orientation plasticity of two models of orientation selectivity, the MFM and MRM, systematically. Although the MFM and the MRM emphasize the feedforward and intracortical mechanisms, respectively, both models contain tuned feedforward input from LGN and intracortical interactions. For the MFM, the feedforward input is well tuned to orientation. The model uses cortical antiphase inhibition to achieve contrast invariance and cortical excitation to boost the tuned activity (Troyer et al., 1998). The cells in this model are simple cells (Teich \& Qian, 2006). For the MRM, the feedforward input is only broadly tuned to orientation and is sharpened by phase-independent recurrent cortical excitation and inhibition in the form of a Mexican hat profile. The model also contains antiphase inhibition for maintaining spatial phase information. We previously used the MRM to produce the full spectrum of simple-to-complex characteristics by varying the relative strengths of antiphase inhibition and recurrent inhibition (please see fig. 8 from Teich \& Qian, 2006). In this paper, we consider plasticity in both a simple cell version and a complex cell version of the MRM. When the antiphase inhibition is strong and recurrent inhibition is weak, the model cells are simple, with an F1:F0 ratio of 1.53. When recurrent inhibition is strong and antiphase inhibition is weak, the resulting model cells have an F1:F0 ratio of 0.89 and are thus complex. Since there is currently no model for orientation plasticity in simple cells, it is particularly interesting to investigate the two simple cell models: the MFM and the simple cell MRM.

Around the trained/adapted orientation, both learning and adaptation reduce a V1 cell's peak response to its preferred ori- entation, although responses to other orientations may increase (Dragoi et al., 2000; Schoups et al., 2001). Our modeling approach is first to modify connections in the models to simulate the peak response reduction and then to examine the consequent changes to tuning curve shapes. The advantage of this approach is that synaptic modification is activity based and thus physiologically plausible; arbitrary modifications of a large number of connections for the sole purpose of fitting the observed tuning curve changes are avoided. We designate the trained/adapted orientation as $0 \mathrm{deg}$. To reduce peak responses around $0 \mathrm{deg}$, we either reduce cortical excitation for cells tuned around $0 \mathrm{deg}$ or reduce both cortical excitation and cortical inhibition but make sure the combined result leads to a peak response reduction for cells tuned around $0 \mathrm{deg}$. In addition, we consider both pre- and postsynaptic rules for modifying intracortical connections: a given connection can be reduced based on the past activity of either the pre- or the postsynaptic cell. Thus, we have four plasticity conditions for intracortical connections: presynaptic reduction of excitation (pre-e), postsynaptic reduction of excitation (post-e), presynaptic reduction of both excitation and inhibition (pre-ei), and postsynaptic reduction of both excitation and inhibition (post-ei). Finally, we also examine a postsynaptic-based reduction of the feedforward excitation (post-f). We do not consider a presynaptic-based modification of the feedforward connections because the presynaptic LGN cells are not orientation tuned. In total, we consider five different forms of plasticity (pre-e, post-e, pre-ei, post-ei, and post-f) in three models (MFM, simple cell MRM, and complex cell MRM).

We first consider a presynaptic-based reduction of intracortical excitation (the pre-e condition). The reduction is maximal for excitatory connections from the presynaptic V1 cell tuned to $0 \mathrm{deg}$ and gradually trails off for other preferred orientations (see Materials and methods). For Fig. 2, the maximal reduction of connection strength (at $0 \mathrm{deg}$ ) is $40 \%$ for the MFM and $4 \%$ for the simple cell MRM and complex cell MRM, which generates an activity reduction at $0 \mathrm{deg}$ of $30 \%$ in the MFM, $25 \%$ in the simple cell MRM, and $24 \%$ in the complex cell MRM. The fact that a $40 \%$ reduction of the connection strengths in the MFM and a $4 \%$ reduction of the connection strengths in the MRM achieve a similar reduction in firing has to do with a quantitative difference between the cortical connection strengths in the two models (please see the methods section of Teich \& Qian, 2006, for a full explanation of the differences between the two models). The Mexican hat cortical connection profile in the MRM (and absent from the MFM) causes a small reduction in cortical excitation to yield a large reduction in the amplitude of orientation tuning curves (please see the methods section of Teich \& Qian, 2003a, for a full explanation of this phenomenon).

Fig. 2 shows that a pre-e reduction has a similar effect in all three models: primarily an amplitude reduction for cells near the trained orientation ("near" cell, panels A1, A2, and A3) and little effect for cells further away from the trained orientation ("far" cell, panels B1, B2, and B3). We find that there is a slight amount of peak shift away from 0 deg for "near" cells in the MFM and simple cell MRM. Even with an activity reduction of $40-45 \%$ at $0 \mathrm{deg}$, the outward peak shift is smaller than $2 \mathrm{deg}$. Another change is a reduction of slope at $0 \mathrm{deg}$. These changes are similar to the changes noted in a pre-e simulation of Felsen et al. (2002) (see Discussion). Compared with the actual tuning curve changes induced by learning and adaptation listed in Introduction, the pre-e condition cannot explain either the learning or the adaptation data completely. Although the MFM and simple cell MRM show two of the adaptation-induced changes (peak shift away from and slope reduction at the adapted orientation), the 

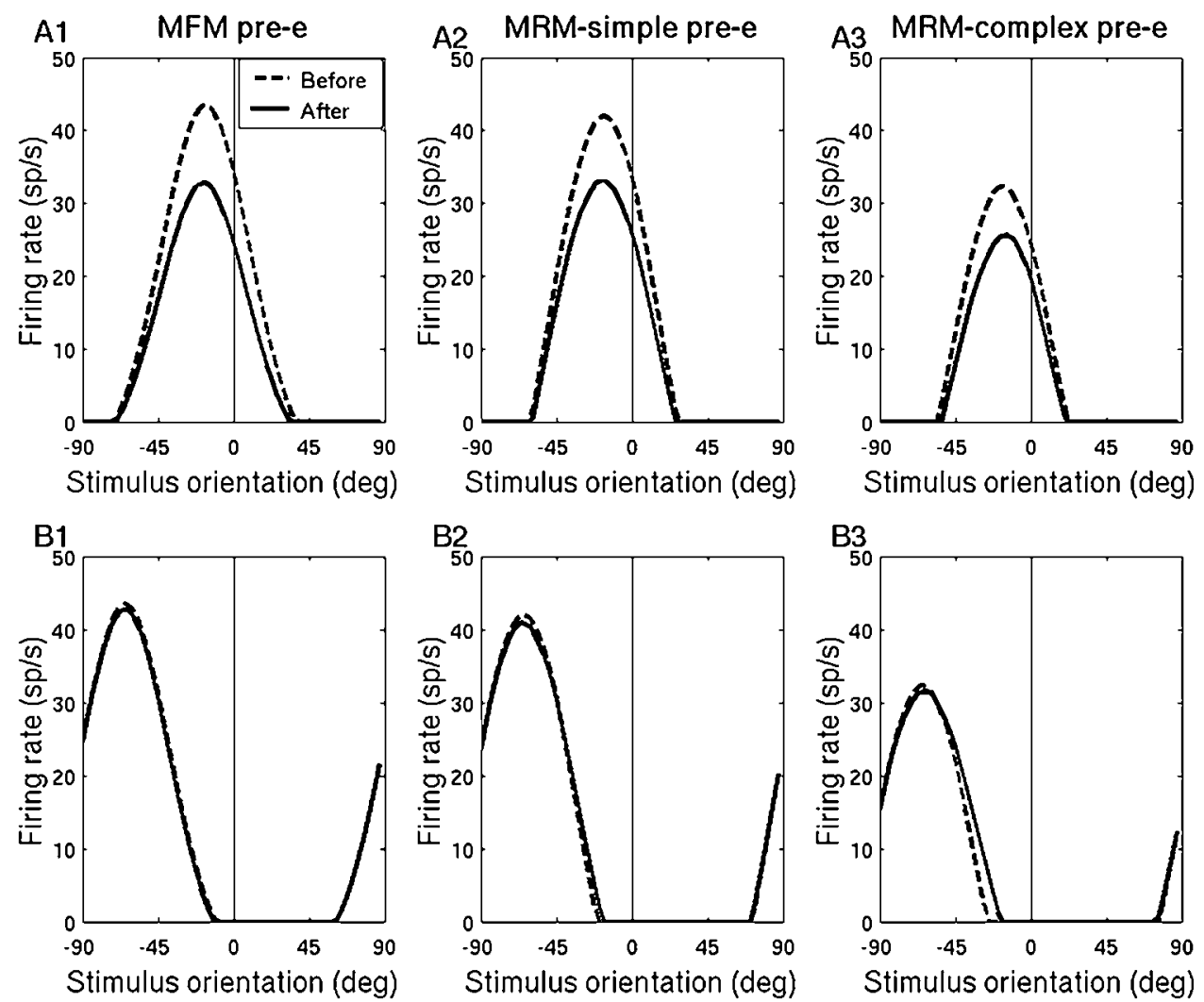

Fig. 2. Tuning curve changes following a presynaptic-based reduction in cortical excitatory connections (pre-e) around 0 deg orientation (vertical line). The three columns show results from the three models (MFM, simple cell MRM, and complex cell MRM), respectively. The top row is for a "near" cell whose preferred orientation is near 0 deg (17 deg away). The bottom row is for a "far" cell whose preferred orientation is far from 0 deg ( 65 deg away). In each panel, the dotted and solid curves are the same cell's tuning curves before and after synaptic modifications.

peak shift (less than $2 \mathrm{deg}$ ) is too small compared with the data (as large as $10 \mathrm{deg}$ ). More importantly, the pre-e condition cannot explain the adaptation-induced response increase on the far flank of the tuning curve. The "near" cells in the complex cell MRM show a slight peak shift toward 0 deg (Fig. 2, A3). However, they do not show a slope increase observed after orientation learning.

We next consider a postsynaptic-based reduction of intracortical excitation (the post-e condition). To generate Fig. 3, the maximal reduction of excitatory connections at $0 \mathrm{deg}$ is $40 \%$ for the MFM, $4 \%$ for the simple cell MRM, and 2\% for the complex cell MRM. These connection reductions generate maximal activity reductions of $31 \%$ for the MFM, 28\% in the simple cell MRM, and $17 \%$ in the complex cell MRM at 0 deg. Note that we use a relatively small connection and activity reduction for the complex cell MRM because the tuning curves in this model are the most plastic (Fig. 3). Both versions of the MRM show narrowing of tuning curves near the 0 deg orientation, sharpening of tuning curve slopes around $0 \mathrm{deg}$ (Fig. 3, A2 and A3), and broadening of tuning curves far from 0 deg (Fig. 3, B2 and B3). These changes agree with the observed tuning curve changes induced by learning (Schoups et al., 2001). We previously found these changes in the complex cells of the RM after a post-e modification (Teich \& Qian, 2003a, 2007). Here, we show that the same changes occur in both the simple cell and the complex cell versions of the MRM. By contrast, the MFM shows only a peak reduction (Fig. 3, A1). This is a theme that will be repeated in the other conditions below; the MRM shows a high degree of plasticity, whereas the MFM shows no such plasticity.
To further quantify these changes, we plot the slope of each tuning curve at $0 \mathrm{deg}$ for each model following the post-e modification (Fig. 4, panels A, B, and C). These three panels show the pretraining orientation tuning curve slopes (dotted lines) and different degrees of excitatory depression (solid lines). We see that cells in both versions of the MRM (panels B and C) have increased their slope at 0 deg after the post-e modification, whereas cells in the MFM have not (panel A). Note that increasing amounts of excitatory depression cause increasing tuning curve slopes in both versions of the MRM, which illustrates the robustness of our results against parametric variations. The plasticity seen in the two versions of the MRM is similar to the findings from the perceptual learning study of Schoups et al. (2001) (panel D). Not only do panels B, C, and D all show an increase in slope at $0 \mathrm{deg}$, but the increase in slope in all three panels is greatest for cells with the largest slope before learning. Also note that even when we depress excitatory connections in the MFM to $10 \%$ of their original strength, so that the maximal activity reduction at $0 \mathrm{deg}$ is as large as $50 \%$, there is still less than $1 \mathrm{deg}$ of maximal peak shifting in the MFM and no slope sharpening (thick solid line in panel A).

Fig. 5 shows the simulation results for a presynaptic-based reduction of both intracortical excitation and intracortical inhibition (the pre-ei condition). The figure is generated with a maximal reduction at $0 \mathrm{deg}$ of $40 \%$ for excitatory and inhibitory connections in the MFM, $40 \%$ for excitatory and $43 \%$ for inhibitory connections in the simple cell MRM, and $20 \%$ for excitatory and inhibitory connections in the complex cell MRM. This generates a maximal 

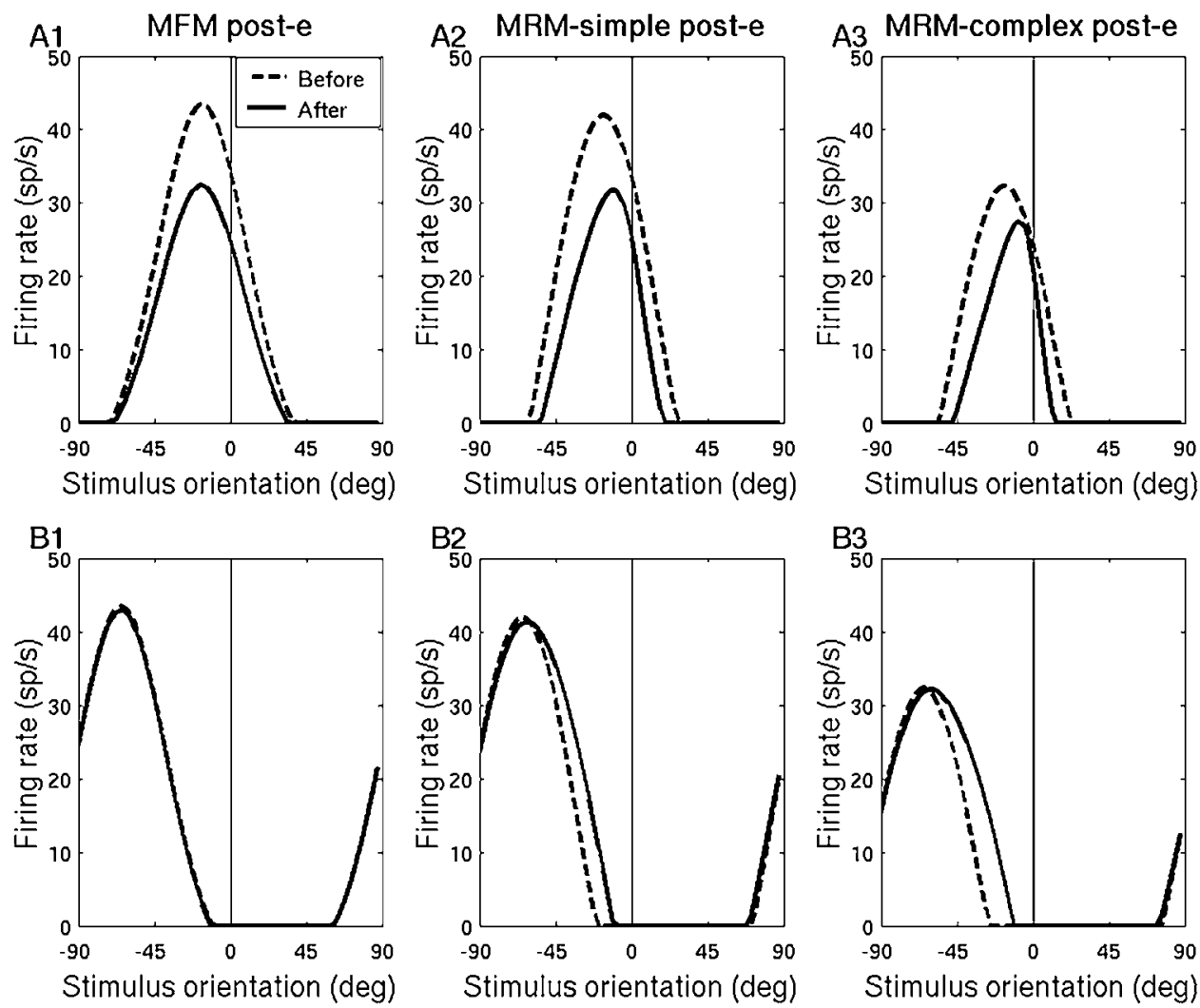

Fig. 3. Tuning curve changes following a postsynaptic-based reduction in cortical excitatory connections (post-e) around 0 deg orientation (vertical line). The format of presentation is identical to that of Fig. 2.

peak activity reduction at $0 \mathrm{deg}$ of $25 \%$ in the MFM, $26 \%$ in the simple cell MRM, and $15 \%$ in the complex cell MRM. Note that unlike in the pre-e and post-e simulations, the pre-ei simulation requires that both recurrent excitation and recurrent inhibition are reduced by a large amount in the MRM, at a level comparable to the MFM. This also applies to the post-ei condition below and has to do with the balance of excitation and inhibition in the MRM (please see the methods section of Teich \& Qian, 2003a, for a full explanation).

In the pre-ei condition, the two versions of the MRM show sharpening and inward skewing of peaks of orientation tuning curves for cells near the 0 deg orientation (Fig. 5, A2 and A3) and broadening of tuning curves for cells whose preferred orientation is further away from 0 deg (Fig. 5, B2 and B3). Thus, this scenario yields results similar to the post-e condition and agrees with the learning-induced tuning curve changes. Again, the MFM shows a different result. The decreased excitation and inhibition cause decreased tuning curve amplitudes and increase responses on the far flanks of the tuning curves of near cells (Fig. 5, A1), but there are no changes on par with the MRM and no changes that closely match the physiology of learning or adaptation.

Note that the MRM has two kinds of inhibitory connections: phase-independent inhibition in the Mexican hat profile and antiphase inhibition (see Materials and methods). The reduction in inhibition in the MRM in Fig. 5 is specifically in the inhibitory connections that form the Mexican hat; connections from antiphase inhibition are not reduced. We have done simulations that reduce the antiphase connections, and we find that this generally does not affect the tuning curve width/slopes or the tuning curve peak location. The reason is that antiphase inhibition in the MRM primarily preserves spatial phase information and inhibits responses to stimuli far from a cell's preferred orientation; responses close to a cell's preferred orientation are primarily inhibited by inhibition from the Mexican hat. Thus, lessening antiphase inhibition in the MRM can moderately increase the response of cells to orientations far from the preferred orientation, similar to the effect seen in the MFM when antiphase inhibition is decreased (Fig. 5, A1). Since reducing antiphase inhibition does not affect the tuning curve width/slopes or tuning curve peak location in MRM cells, we will not include these simulations here for the sake of brevity.

Fig. 6 shows the results of a postsynaptic-based reduction of both intracortical excitation and intracortical inhibition (the post-ei condition) for the three models. The figure is generated with a maximal reduction at $0 \mathrm{deg}$ of $40 \%$ for excitatory and inhibitory connections in the MFM, $40 \%$ for excitatory and $43 \%$ for inhibitory connections in the simple cell MRM, and $42 \%$ for excitatory and $40.5 \%$ for inhibitory connections in the complex cell MRM. This generates a maximal peak activity reduction at $0 \mathrm{deg}$ of $26 \%$ in the MFM, 22\% in the simple cell MRM, and 29\% in the complex cell MRM. For both versions of the MRM, cells with preferred orientations near 0 deg show reduced slopes of orientation tuning curves around $0 \mathrm{deg}$ (referred to as "near broadening" below), their tuning curve peaks skew away from $0 \mathrm{deg}$, and they have increased responses on the flank of the tuning curve that face away from $0 \mathrm{deg}$. Cells with preferred orientations further away from 0 deg have modest sharpening of their tuning curves (referred to as "far narrowing" below). All these changes agree with the adaptation data (Dragoi et al., 2000, 2002; Felsen et al., 2002). We previously found these changes for the complex cells in the RM in a post-ei modification (Teich \& Qian, 2003a). Here, we show that the same changes occur in both simple cell and complex cell versions of the 


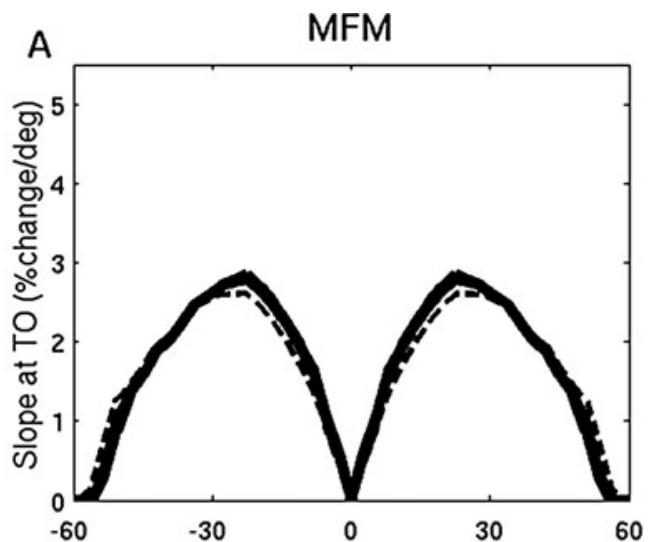

Preferred orientation-trained orientation (deg)

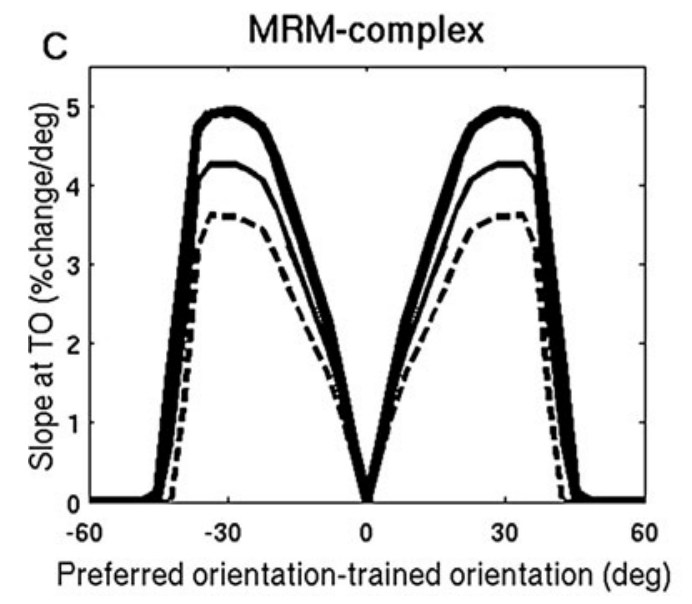

Preferred orientation-trained orientation (deg)

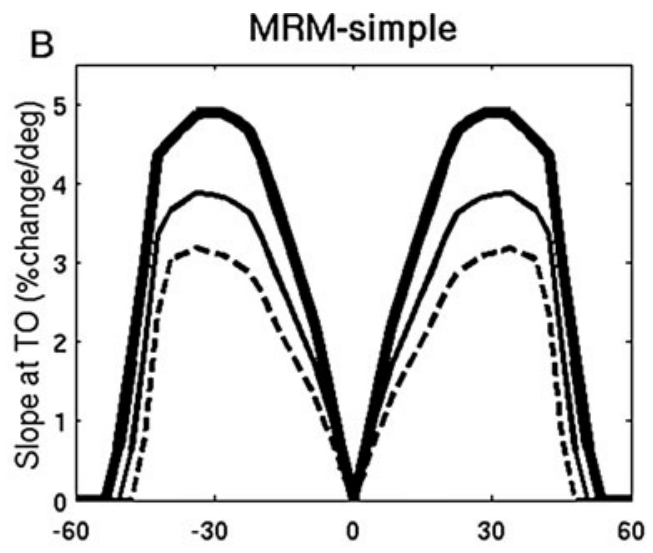

Preferred orientation-trained orientation (deg)

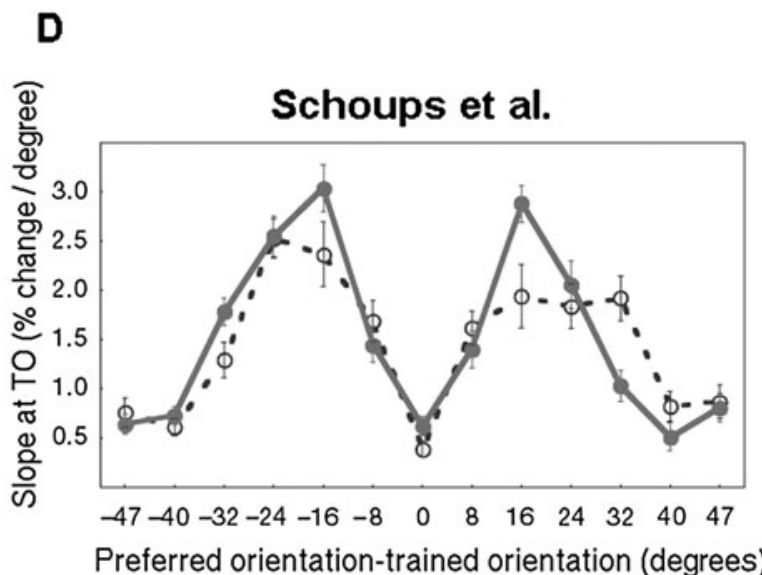

Fig. 4. Comparison between post-e simulations and learning data. Panels (A), (B), and (C) show the results for the MFM, simple cell MRM, and complex cell MRM, respectively. For each model, the slope of every V1 cell's tuning curve at 0 deg is plotted against the difference between its preferred orientation and the trained orientation $(0 \mathrm{deg})$ both before (dotted line) and after (solid lines) a post-e modification. For the MFM in panel (A), there is no increase in slope around the trained orientation for either a $40 \%$ (thin solid line) or a 90\% (thick solid line) maximal reduction in excitation around 0 deg. In panel (B), the simple cell MRM shows increasing slope around the trained orientation after a $2 \%$ (thin line) and $4 \%$ (thick line) maximal reduction in excitation at $0 \mathrm{deg}$. The same holds for the complex cell MRM in panel (C) after a $0.5 \%$ (thin line) and $1 \%$ (thick line) maximal reduction in excitation at 0 deg. Panel (D) shows the data taken from Schoups et al. (2001) with permission. TO stands for trained orientation, which is 0 deg for the simulations.

MRM. Furthermore, we show that in contrast, the MFM does not show robust changes in the post-ei condition that can account for either the learning or the adaptation data.

To further show that the simulations with the MRM are in line with the experimental data, we plot peak shift in comparison to a figure from Dragoi et al. (2000) that shows average peak shift after adaptation (Fig. 7). Fig. 7 shows that both versions of the MRM generate peak shift that is in the same range as the data from Dragoi et al. (2000). Note that the simulation panels (A, B, and C) each shows two different simulations with reduced excitatory and inhibitory connections (dotted and solid lines), which show the robustness of our results against parametric variations. Both versions of the MRM demonstrate a similar degree and distribution of peak shifting with different amounts of activity reduction, as long as excitatory and inhibitory connections are both reduced by about the same amount. In contrast, the MFM does not show robust changes that can account for the data. Again, even if we depress the connections in the MFM further so that excitatory and inhibitory connections are reduced to $10 \%$ of their original strength and the maximal activity reduction is as large as $40 \%$, there is still less than a degree of maximal peak shifting (panel A, dotted line). Note that the MRM simulations (panels B and C) and the data of Dragoi et al. (2000) in panel D show the largest peak shift at different orientations. We find that the MRM can match the data if we let the standard deviation of the recurrent excitation and inhibition be 25 and $37 \mathrm{deg}$, respectively, so that the Mexican hat has a slightly narrower profile. However, we did not use this set of parameters as our standard set because it would be more difficult to visually appreciate the broadening and narrowing of tuning curves, even though they are present.

Finally, we also consider a postsynaptic reduction of feedforward excitation in the models (the post-f condition). When we previously modeled learning and adaptation in the RM (Teich \& Qian, 2003a), we found that the post-f condition was similar to the post-e condition in generating tuning curve changes that match the learning data. This is not surprising because in both cases, a postsynaptic V1 cell has all excitatory connections (either thalamic or cortical) to it reduced by a common factor based on the cell's past activity. We did not report the details previously, but we do so here for the MFM and MRM for completeness. Since the MFM assumes that sharp V1 orientation 

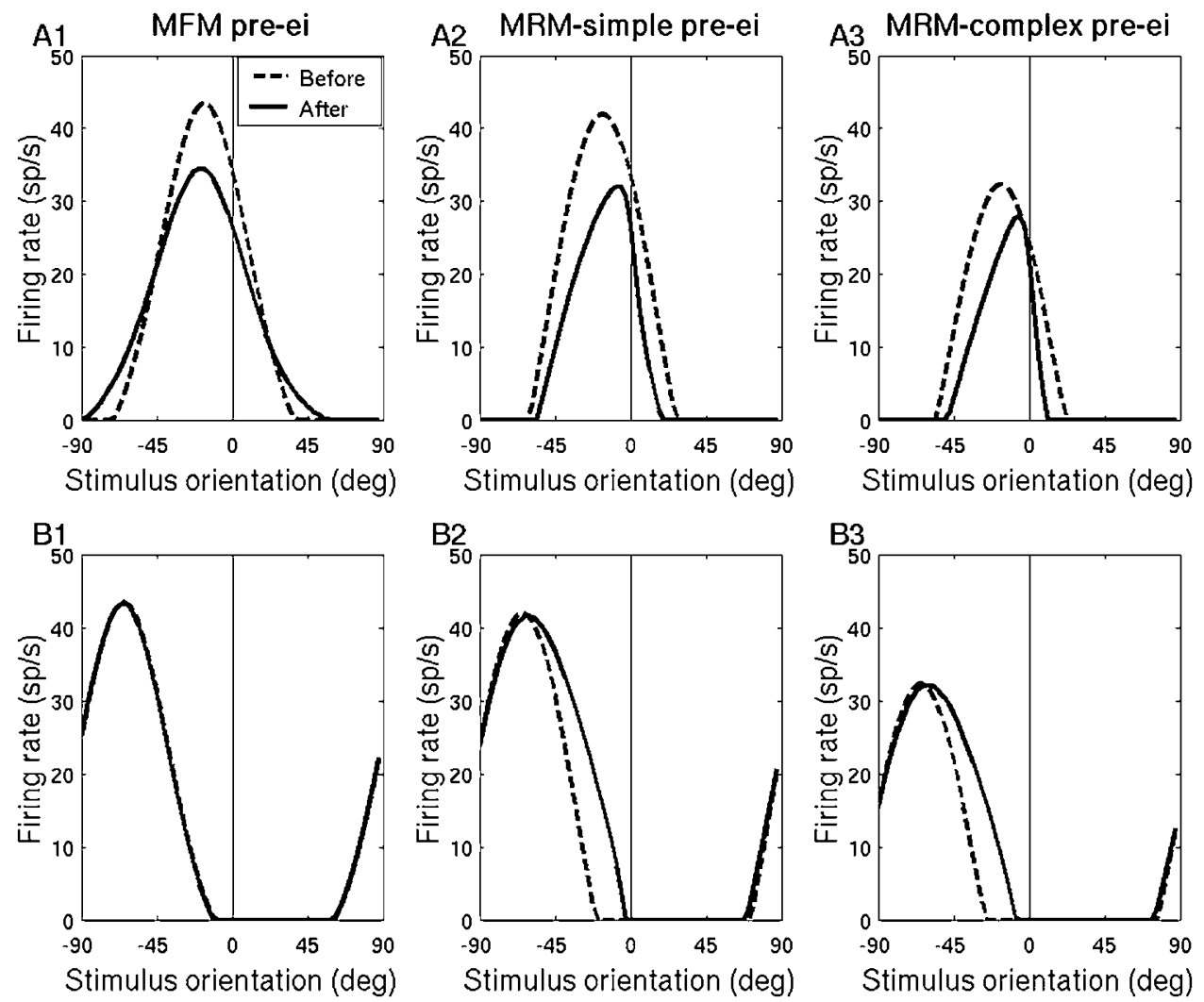

Fig. 5. Tuning curve changes following a presynaptic-based reduction in cortical excitatory and inhibitory connections (pre-ei) around 0 deg orientation (vertical line). The format of presentation is identical to that of Fig. 2.

tuning originates from the feedforward input, it is interesting to examine how a post-f modification affects tuning curves in this model. Fig. 8 is generated with a maximal reduction at 0 deg of $30 \%$ for feedforward connections in the MFM, 20\% for feedforward connections in the simple cell MRM, and $10 \%$ for feedforward connections in the complex cell MRM. This generates a maximal peak reduction at $0 \mathrm{deg}$ of $34 \%$ in the MFM, $22 \%$ in the simple cell MRM, and $11 \%$ in the complex cell MRM. As in the post-e condition in Fig. 3, the MFM shows primarily an amplitude reduction. However, the MRM for both simple and complex cells demonstrates inward skewing of tuning curve peaks for cells near the 0 deg orientation, broadening of tuning curves for cells farther away from $0 \mathrm{deg}$, and sharpening of the tuning curve slope around $0 \mathrm{deg}$. Note that under the post-f condition, a small degree of amplitude reduction in the complex cell MRM produces robust plasticity in tuning curve shape. In general, we find that the complex cell MRM shows a stronger degree of plasticity than the simple cell MRM (cf., Figs. 3,5 , and 6). This is due to the fact that nearly all the inhibition in the complex cell MRM comes from the Mexican hat cortical interaction profile, whereas much less of the overall inhibition in the simple cell MRM comes from this profile (a larger portion of inhibition comes from antiphase inhibition). We find that the Mexican hat interaction profile is a key component of plasticity (see below).

We have demonstrated that both the simple cell and the complex cell versions of the MRM (and the original RM) show rich plasticity of orientation tuning that can match learning and adaptation data, while the MFM does not. We now provide an intuitive analysis of what properties inherent in the MRM and RM and absent from the MFM are critical for robust plasticity. We have identified two such properties. The first is the Mexican hat intracortical interaction profile. The second is a broadly tuned feedforward input. To illustrate the dependence of plasticity on these properties, we focus on one specific phenomenon from the above simulations: the near broadening (Fig. 6, A2 and A3) and far narrowing (Fig. 6, B2 and B3) in the MRM for the post-ei condition that matches the adaptation-induced plasticity. Why do cells near 0 deg (the adapted orientation) broaden their tuning curves? To understand this behavior, we must consider what happens to the Mexican hat intracortical interaction profile after a post-ei modification in the MRM.

In Fig. 9, panel A, the dashed curve shows the baseline Mexican hat intracortical connection profile to a postsynaptic V1 cell tuned to $0 \mathrm{deg}$ before modification. This profile is the difference between the excitatory and inhibitory connections the cell receives. It sharpens a broadly tuned feedforward input by boosting responses near 0 deg and suppressing responses at far orientations (dashed tuning curve of panel B). Now consider the post-ei modification of the connections. Since both the excitatory and the inhibitory components of the Mexican hat are reduced, the strengths of both net excitation from near orientations and net inhibition from far orientations to the cell are reduced, resulting in a scaled down Mexican hat (solid curve of panel A). The reduced net near-excitation decreases the tuning curve amplitude at its peak, and the reduced net far-inhibition broadens the flanks of the tuning curves (solid curve of panel B). Once this phenomenon of near broadening is understood, it becomes clear why in Fig. 6 tuning curves narrow for cells whose preferred orientation is far from $0 \mathrm{deg}$. Because of the Mexican hat interaction profile, cells near 0 deg inhibit cells far from 0 deg. Since cells near 

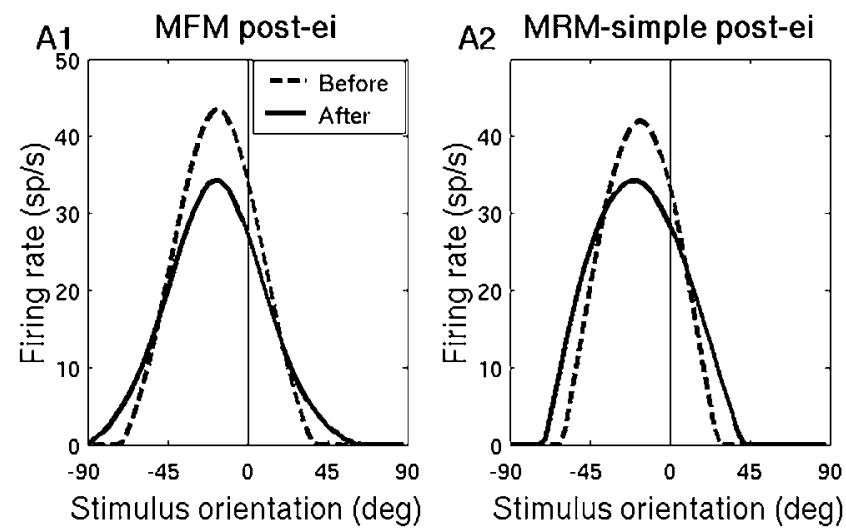

Stimulus orientation (deg)

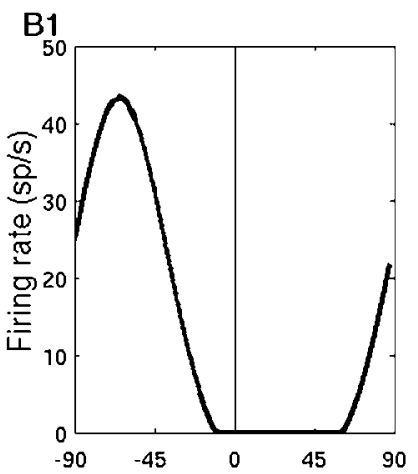

Stimulus orientation (deg)
B2

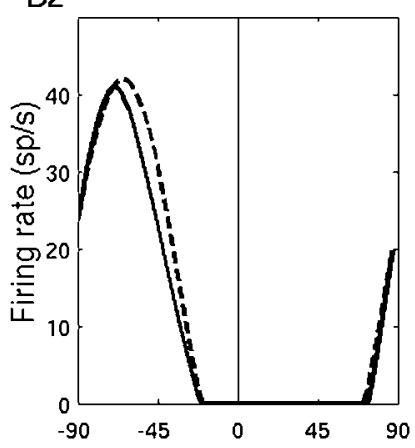

Stimulus orientation (deg)

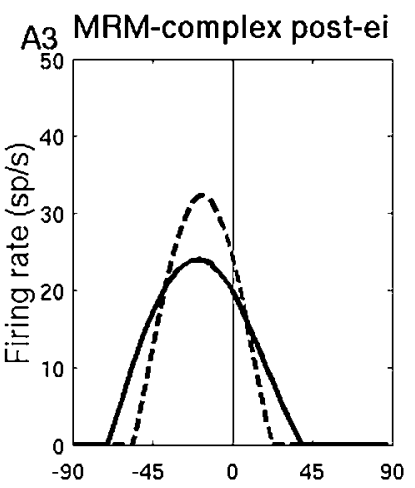

Stimulus orientation (deg)

B3

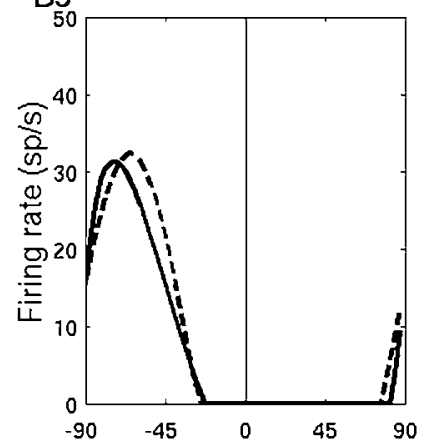

Stimulus orientation (deg)

Fig. 6. Tuning curve changes following a postsynaptic-based reduction in cortical excitatory and inhibitory connections (post-ei) around $0 \mathrm{deg}$ orientation (vertical line). The format of presentation is identical to that of Fig. 2.

0 deg now have expanded flanks, there is more overlap between their tuning curves and far cells' tuning curves. Thus, they provide more side inhibition to far cells, and since far cells do not have modified Mexican hats that might cause broadening, the net effect is for far cells to narrow their tuning.

The above reasoning also explains the lack of an effect on far cells in the MFM under the post-ei modification (Fig. 6). In Fig. 6, even though the near cell in panel A1 does not really broaden, the reduction in inhibition does cause the cell to fire at more distant orientations. However, because of the lack of a Mexican hat, this change does not affect the width of the far cell in panel B1.

In addition to the Mexican hat interaction profile, a broadly tuned input is also critical for the near broadening discussed above. In order for a reduction in net flank inhibition to yield a broader tuning curve, there needs to be some input at flank orientations that is normally being inhibited. A useful analogy is to think of a sculptor making a statue out of rock. If one starts with a large piece of rock, then chiseling away less stone can produce a wider statue. However, if the sculptor is given a piece of rock that is already in the narrow shape of the final statue, it is impossible to produce a wider statue by modifying the sculpting technique. The top row of Fig. 10 shows the simple cell MRM results in a post-ei scheme for both a broadly tuned and a narrowly tuned feedforward input. The characteristic near broadening (panel A1) is nearly lost when the feedforward input is already sharply tuned for orientation (panel A2). Fig. 10 also compares broad and narrow feedforward inputs for the post-e condition (bottom row). Here, the near sharpening of the slope at $0 \mathrm{deg}$ (panel B1) is diminished when the feedforward input is already sharply tuned (panel B2).
We find that peak shift of tuning curves also depends on the Mexican hat profile and a broadly tuned feedforward input. Fig. 10 shows that in the simple cell MRM, the peak skewing away from 0 deg under the post-ei condition and the skewing toward 0 deg under the post-e condition are both reduced for a sharply tuned feedforward input. This partially explains why the MFM, which assumes a sharply tuned feedforward input, does not show significant peak shift in all simulation conditions, while the MRM, which normally assumes a broadly tuned feedforward input, does. In general, a broadly tuned feedforward input allows for a greater range of possibilities for the final tuning curve to be shaped by both the excitation and the inhibition a cell receives from other cortical cells. Less inhibition from cells on one side of the tuning curve will pull the peak in this direction; less excitation will cause it to be pulled in the opposite direction.

\section{Discussion}

We have systematically investigated orientation plasticity of a feedforward model (the MFM) and a RM (the MRM). Despite their names, both models contain a feedforward input and recurrent cortical interactions. However, the MFM assumes well-tuned feedforward input not sharpened further by cortical interactions, while the MRM assumes a broadly tuned feedforward input sharpened by cortical interactions. The MFM is for simple cells. The MRM, on the other hand, can display a continuum of simpleto-complex characteristics so we use both a simple cell version and a complex cell version of the MRM. We consider plausible synaptic modification rules based on the past activities of either pre- or postsynaptic cells and we reduce either excitatory connections 

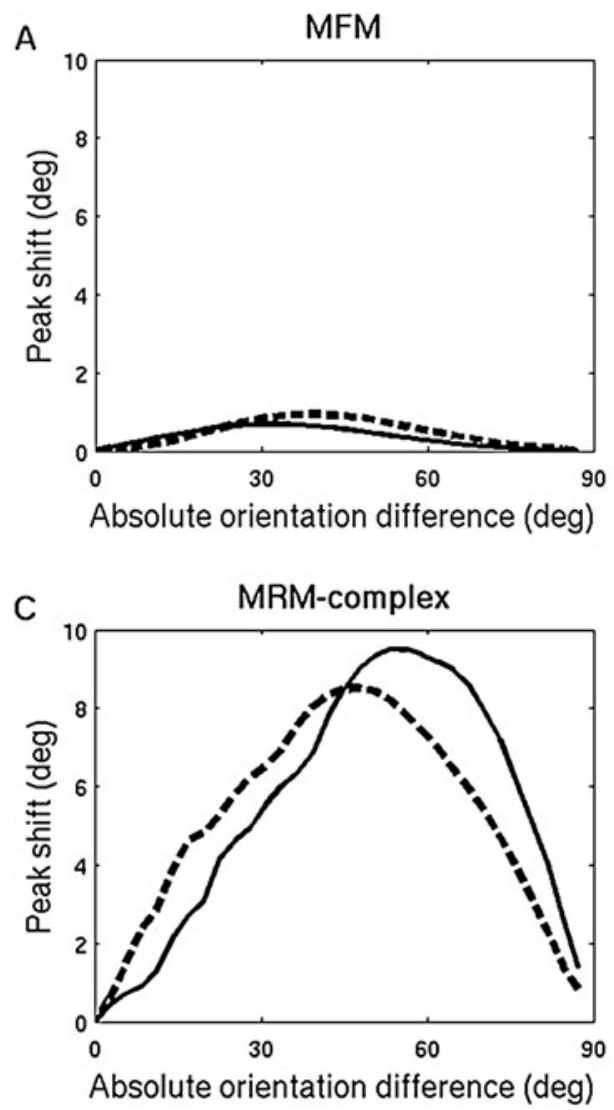

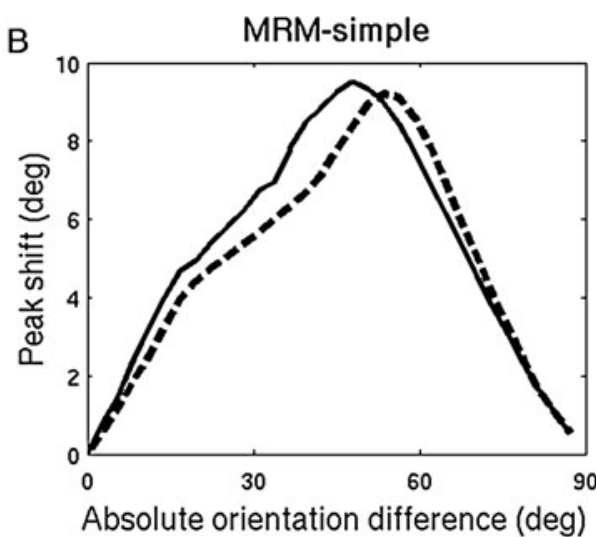

D

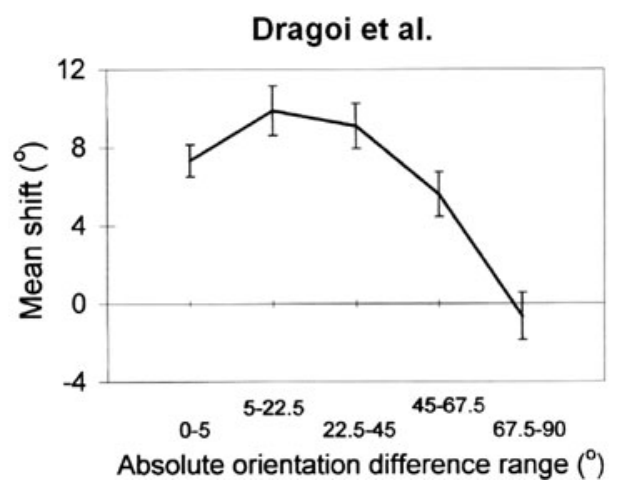

Fig. 7. Comparison between post-ei simulations and adaptation data. Panels (A), (B), and $(\mathbf{C})$ show the results for the MFM, simple cell MRM, and complex cell MRM, respectively. Each of these three panels shows two different simulations. For each model, the peak shift of every V1 cell's tuning curve generated by a post-ei modification is plotted against the difference between its preferred orientation and the adapted orientation ( $0 \mathrm{deg}$ ). In panel (A), the MFM shows minimal peak shifting after either a $40 \%$ reduction in excitation and inhibition (solid line) or a 90\% reduction in excitation and inhibition (dotted line) at 0 deg. Panel (B) shows the simple cell MRM after a $40 \%$ reduction in excitation and a $44 \%$ reduction in inhibition (solid line) and a $60 \%$ reduction in excitation and a $63 \%$ reduction in inhibition (dotted line). Note that both manipulations cause a similar distribution and degree of peak shifting. The same phenomenon is seen in panel (C), which shows the complex cell MRM after a $42 \%$ reduction in excitation and a $40.5 \%$ reduction in inhibition (solid line) and a $22 \%$ reduction in both excitation and inhibition (dotted line). Panel (D) shows the data taken from Dragoi et al. (2000) with permission.

alone or both excitatory and inhibitory connections. We find that the MFM does not demonstrate plasticity that can account for the changes seen in learning or adaptation experiments (Dragoi et al., 2000, 2001; Schoups et al., 2001; Felsen et al., 2002), whereas the MRM can explain these changes for both simple and complex cells. Specifically, the MRM reproduces the adaptation data with a postsynaptic-based reduction of intracortical excitation and inhibition (post-ei). The same model reproduces the learning data with a postsynaptic-based reduction of intracortical excitation (post-e), a postsynaptic-based reduction of feedforward excitation (post-f), or a presynaptic-based reduction of intracortical excitation and inhibition (pre-ei). Thus, the adaptation data place a stronger constraint on synaptic modification than the learning data do. Two features of the MRM, that are absent from the MFM, are found to be critical for robust plasticity: a Mexican hat intracortical connection profile and a broadly tuned feedforward input. Both these features are also found in the original RM for complex cells and explain plasticity in that model as well (Teich \& Qian, 2003a). This result is consistent with the experimental finding that plasticity is weaker in isoorientation domains than in pinwheel centers of V1 (Dragoi et al., 2001), as cells in iso-orientation domains may receive cortical inputs from only a restricted range of preferred orientations, and the resulting weak Mexican hat profile may lead to weak plasticity regardless of the breadth of the feedforward tuning. Our study is the first that reproduces the observed orientation plasticity in a simple cell model. Moreover, our exhaustive exploration of different synaptic modification schemes in the MFM and the MRM provides a fairly complete picture of orientation plasticity of the models and establishes a basis for using plasticity to evaluate feedforward and recurrent mechanisms of orientation selectivity.

There is currently no consensus over how orientation selectivity originates in V1, and many specific assumptions in the models are debatable (see Teich \& Qian, 2006, for a recent discussion). Our systematic investigation of orientation plasticity in the MFM and the MRM offers a new opportunity for addressing the issue. For example, Ferster et al. have provided some of the strongest evidence for a feedforward mechanism of orientation selectivity for the simple cells in the input layer of cat V1 (Ferster et al., 1996; Chung $\&$ Ferster, 1998). Based on our work, their conclusion implies that these input-layer cells should not show much orientation plasticity other than a reduction of peak responses after learning or adaptation. In contrast, Dragoi et al. (2000) found similar orientation plasticity across all layers of cat V1. If some of their recorded cells with strong plasticity were input-layer simple cells, our work predicts that these 

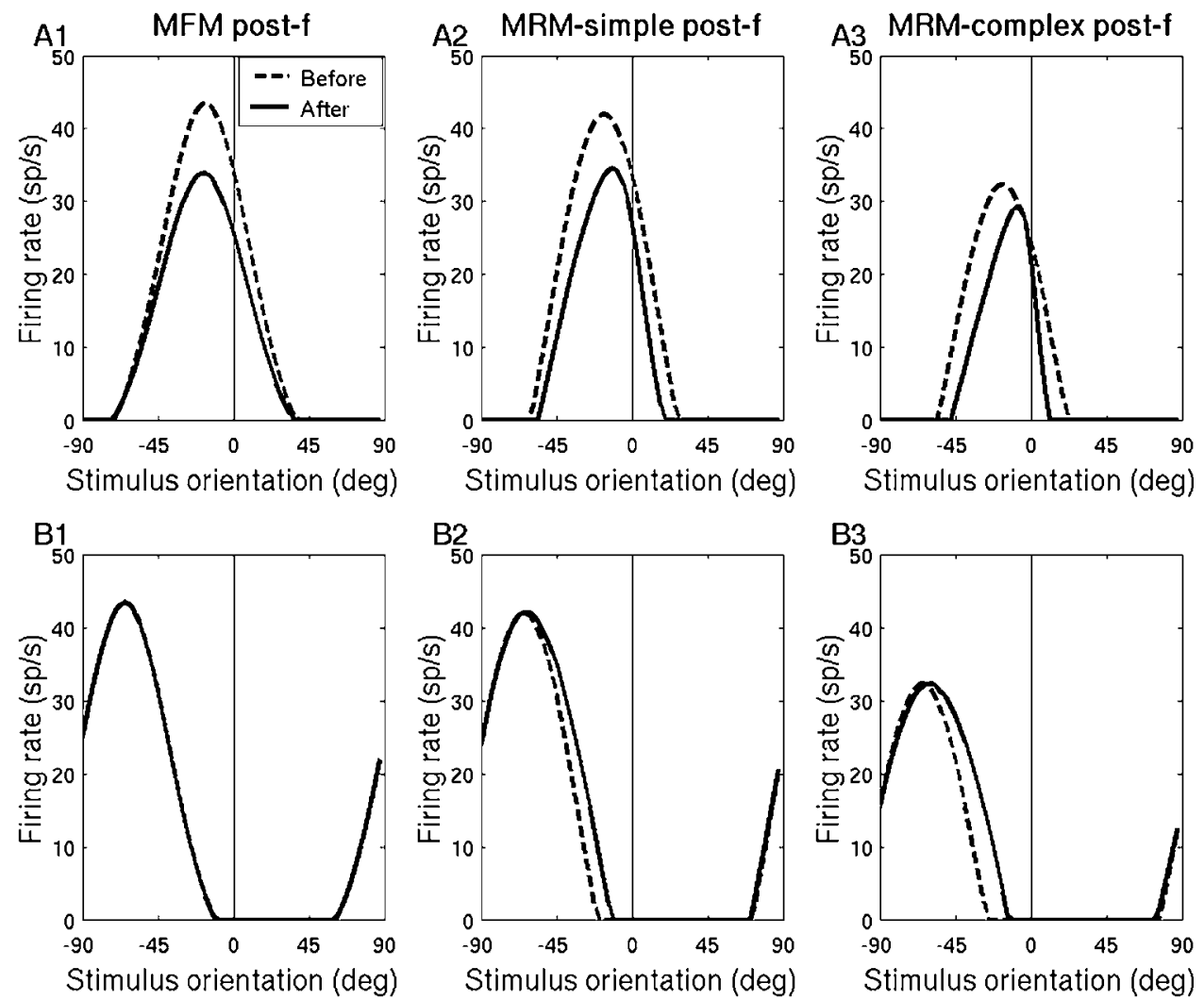

Fig. 8. Tuning curve changes following a postsynaptic-based reduction in feedforward excitatory connections (post-f) around 0 deg orientation (vertical line). The format of presentation is identical to that of Fig. 2.

cells must use a recurrent mechanism to generate orientation selectivity. Clearly, to distinguish between different proposals for the origin of orientation selectivity, future experiments could focus on orientation plasticity of input-layer V1 cells. If these simple cells show no plasticity beyond a peak response reduction, then they are better described by a feedforward mechanism such as the MFM. Otherwise, a recurrent mechanism like the simple cell MRM is a better choice. One could also compare the degree of cortical sharpening of feedforward tuning with the degree of orientation plasticity in the same V1 cells and see whether they are positively correlated as predicted by our study. A related observation is that orientation tuning width in at least some simple cells changes with the spatial frequency of a stimulus (Vidyasagar \& Siguenza, 1985). This observation is better explained by a feedforward model because in a RM, the tuning width is largely determined by intracortical interactions and is not very sensitive to stimulus spatial frequency (Ferster \& Miller, 2000; Teich \& Qian, 2006). Therefore, it would also be interesting to compare the degree of the spatial frequency

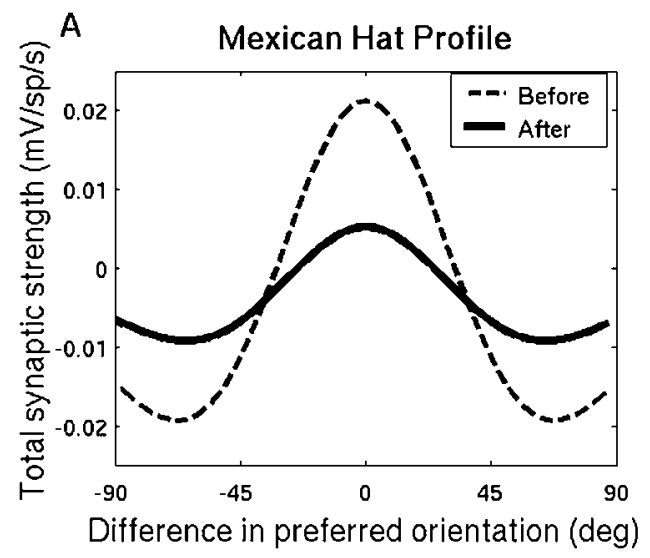

B

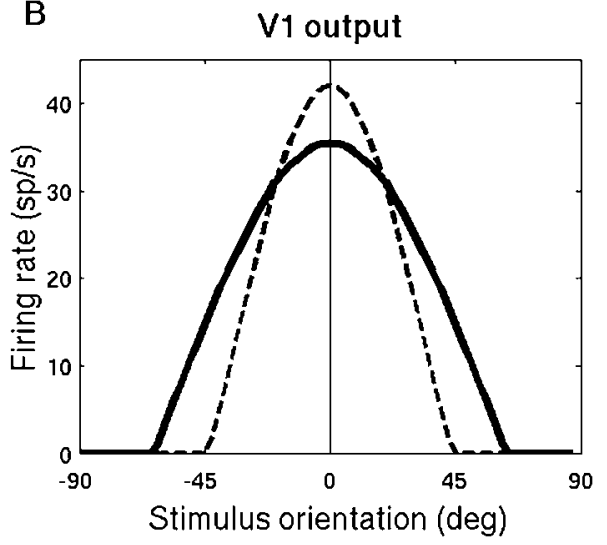

Fig. 9. An explanation for near broadening in the MRM after a post-ei modification. Dotted and solid curves show results before and after the modification, respectively. (A) The Mexican hat profiles for a V1 cell tuned to $0 \mathrm{deg}$. The profile shows the net connection strengths this cell receives from cells tuned to other orientations. (B) The orientation tuning curves of the cell. 

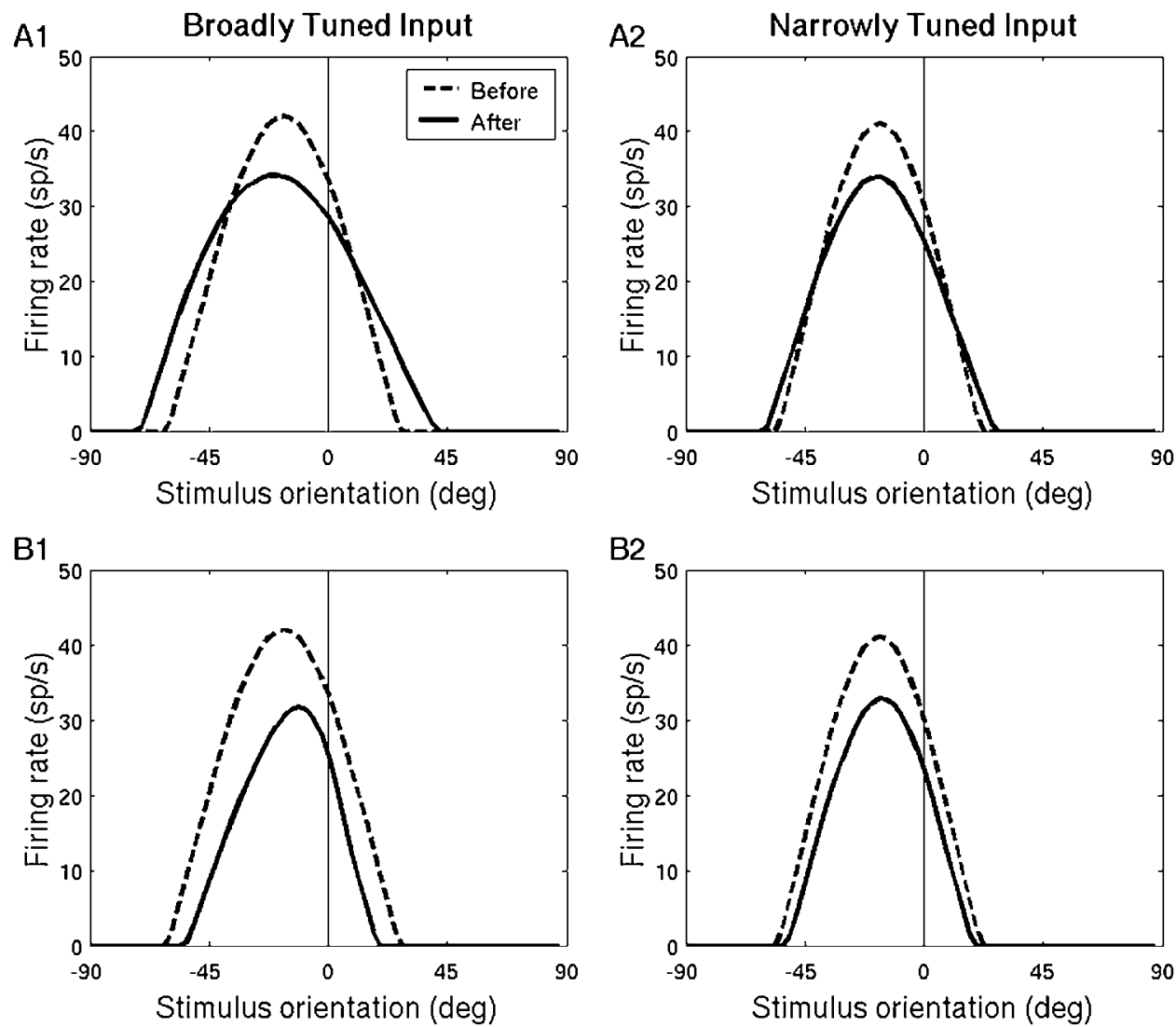

Fig. 10. A broadly tuned input is required for robust plasticity in the MRM (simple). In panel (A1), we see a near cell (17 deg away) for the post-ei condition (as in Fig. 6). In panel (A2), this same cell is now receiving a much more sharply tuned input, identical to the feedforward input in the MFM (aspect ratio = 4.54). Panels $(\mathbf{B 1})$ and $(\mathbf{B 2})$ illustrate the same exercise for the post-e condition (as in Fig. 3). The dotted curves are the tuning curves before modification, and the solid curves are the tuning curves after modification.

dependence of tuning width with the degree of orientation plasticity in the same V1 simple cells and see whether they are negatively correlated. Finally, our simulations with the two versions of the MRM predict that although both simple and complex cells can show similar plastic behavior, the latter should display a stronger degree of plasticity than the former.

Our work predicts that orientation plasticity in both simple and complex cells follows the same mechanism. Although this prediction has not been directly tested, many studies suggest that simple and complex cells do adapt similarly. Movshon and Lennie (1979) found that simple and complex cells in cat V1 show similar shift of their contrast response functions after grating adaptation (although complex cells show somewhat more reliable spatial frequency selective adaptation). This basic finding was confirmed by others in cats (Ohzawa et al., 1982, 1985; Dean, 1983) and monkeys (Sclar et al., 1989). von Der Heydt et al. (1978) showed in cats that adaptation of both simple and complex cells depends on whether the adapting grating is drifting in a cell's preferred or null direction, and Bonds (1991) showed that contrast adaptation can occur even after very brief exposures $(50 \mathrm{~ms})$ in both simple and complex cells in cats. Others have looked closely at adaptation in simple and complex cells and found subtle differences. Maffei et al. (1973) showed that simple cells in cat V1 take longer than complex cells to recover from adaptation, and Albrecht et al. (1984) confirmed this finding in cats while also showing that simple cells are more resistant to adaptation than complex cells. However, Hammond et al. (1988) showed that cat V1 simple and complex cells have roughly the same susceptibility to adaptation, while special complex cells (optimal response to short contours) are generally resistant to adaptation, and that contrary to earlier reports, the time course of adaptation and recovery is shorter in simple cells than in complex cells. One possible explanation for the discrepancy in the literature is that there is far more variability in the response of complex cells to adaptation than simple cells. Several studies have shown that simple cells in cat V1 show significant direction-selective adaptation to a drifting grating and generally have lower direction selectivity after adapting to a grating in their preferred orientation and direction (Marlin et al., 1988; Giaschi et al., 1993). Complex cells, however, are far more variable, and some even increase direction selectivity following preferred direction adaptation.

Regardless of simple/complex types and cortical layers, our work predicts that cells showing strong plastic properties as listed in the Introduction must receive a broadly tuned feedforward input (either from LGN or from poorly tuned V1 cells) sharpened by Mexican hat intracortical interactions. Of course, we cannot rule out the possibility that a new model without these features may be proposed in the future to explain all the observed orientation plasticity. However, our intuitive explanation in Results on why these features are necessary makes such a possibility unlikely.

The existence of Mexican hat intracortical interactions is also controversial and has been discussed in detail in our previous publications (Teich \& Qian, 2003a, 2006). Here, we just note that the inhibitory connection profile only needs to be slightly wider than the excitatory connection profile to generate a net Mexican hat 
for the RMs to produce robust plasticity. A related point is that although the MFM also uses recurrent cortical excitation to boost responses around the preferred orientation and cortical inhibition to suppress contrast-dependent DC responses (i.e. the untuned component) from nonpreferred orientations, it does not have the same Mexican hat as in the RMs. The reason is that cortical excitation and inhibition in the MFM are phase dependent, as well as orientation dependent. Consequently, there is a temporal separation of excitatory and inhibitory input a cell receives (Troyer et al., 1998), and one cannot simply subtract them to get a net connectivity profile. Finally, since both the MFM and the MRM contain recurrent intracortical excitation, it is not accurate to conclude that recurrent cortical interactions lead to robust plasticity. What is important is whether the recurrent connections form a Mexican hat to sharpen broadly tuned feedforward input.

Our conclusion that the MRM is superior to the MFM for modeling orientation plasticity is consistent with previous studies that examined plasticity (Felsen et al., 2002; Teich \& Qian, 2003a). In particular, Felsen et al. (2002) compared a feedforward model with a RM and concluded that the RM was better at producing a repulsive shift of the tuning peak induced by adaptation. However, they used a presynaptic-based modification of cortical excitation (the pre-e condition in this paper) in the RM. As shown in their simulations and our Fig. 2, such a modification scheme can only produce a very small peak shift and cannot explain other aspects of adaptation-induced changes (Dragoi et al., 2000). We find that a postsynaptic-based modification of both cortical excitation and cortical inhibition is needed to produce a large enough peak shift (Fig. 6) and to explain all the adaptation-induced changes (Figs. 6 and 7). Also note that the feedforward model implemented by Felsen et al. (2002) does not contain intracortical excitation that is present in the full version of the MFM (Troyer et al., 1998) as suggested by anatomical (Peters \& Payne, 1993) and physiological experiments (Ferster et al., 1996; Chung \& Ferster, 1998). When such intracortical excitation is included, we show that a feedforward model (the MFM in this paper) also produces a small repulsive peak shift under the pre-e condition but not other adaptation-induced changes (Fig. 2). Therefore, the current study strengthens Felsen et al.'s (2002) conclusion by demonstrating that the feedforward and RMs are best distinguished by systematically examining all plausible modification schemes and taking into account all plastic changes induced by learning and adaptation experiments. Finally, the RM implemented by Felsen et al. (2002) is atypical in that it used a welltuned feedforward input not sharpened by recurrent cortical connections and used a recurrent connection profile with diminished side inhibition. We show in this paper that to explain all the observed plasticity, it is not sufficient to introduce recurrent interactions, which are present in both the MFM and the MRM. Rather, the recurrent connections must form a Mexican hat (with strong side inhibition) that sharpens broadly tuned feedforward input.

In our previous work of modeling orientation plasticity (Teich \& Qian, 2003a), we also considered perceptual implications of the tuning curve changes induced by learning and adaptation. We found that while the learning-induced tuning changes can explain the improved orientation discriminability around the trained orientation, the adaptation-induced tuning changes cannot explain the perceptual consequences of adaptation. Orientation adaptation generates at least two perceptual effects. The first effect is an improvement of orientation discriminability at the adapted orientation as if adaptation is a transient form of learning (Regan \& Beverley, 1985). Whereas the increased tuning slope at the trained orientation explains the improved discriminability for learning, the reduced slope at the adapted orientation cannot explain the same perceptual improvement (Teich \& Qian, 2003a). The second effect is the wellknown tilt aftereffect: After adaptation to a given orientation, nearby orientations appear to be repelled away from the adapted orientation (Gibson, 1933; Wolfe, 1984; Greenlee \& Magnussen, 1987). The tilt aftereffect indicates that the population response of cells preferring different orientations has a repulsive skewing from the adapted orientation. It has long been recognized that the activity reduction at the adapted orientation produces such a repulsive skewing in the population response (Sutherland, 1961; Gilbert \& Wiesel, 1990). However, the repulsive skewing of the tuning curve induced by adaptation means an attractive skewing of the population response (see Appendix 2 for a simple explanation), the opposite of the tilt aftereffect (Gilbert \& Wiesel, 1990; Yao \& Dan, 2001; Teich \& Qian, 2003a). When we plotted the population response from our adaptation simulations, we found that these two opposite "forces" largely cancel each other resulting in little skewing of the population response (Teich \& Qian, 2003a). We concluded that adaptation physiology cannot explain adaptation psychophysics. In contrast, our learning simulation predicts an attractive skewing of the tuning curve peaks and thus a repulsive shift of the population response. So adaptation psychophysics can be well explained by our learning simulations. We thus raised the possibility that adaptation may lead to learning-like tuning curve changes in alert animals or in a later cortical area. Interestingly, Kohn and Movshon (2004) subsequently found that motion adaptation induces tuning curve changes in area MT (middle temporal) of anesthetized monkeys in precisely the matter predicted by our orientation learning simulation. All the main aspects of their data, such as narrowing of tuning curves near the adapted orientation and skewing of tuning curve peaks toward the adapted orientation, are predicted by our learning simulations (Teich \& Qian, 2003a).

Jin et al. (2005) proposed an interesting alternative to reconcile the V1 physiology of orientation adaptation with the tilt aftereffect. These authors did a quantitative analysis of the physiological data from Dragoi et al. (2000) and found that the activity reduction alone would produce too large a tilt aftereffect. After including the canceling effect of the repulsive peak skewing of the tuning curves, the net tilt aftereffect agrees quantitatively with human psychophysical measurements (Clifford et al., 2000). However, one possible problem with their proposal is that in Dragoi et al. (2000), the activity reduction happens first, within $10 \mathrm{~s}$ of adaptation, while the repulsive skewing of tuning curves occurs later and gradually increases as the adaptation time is increased up to $10 \mathrm{~min}$. Thus, Jin et al.'s (2005) model predicts that the tilt aftereffect is most pronounced at $10 \mathrm{~s}$, when there is a great degree of activity reduction but no repulsive shift of tuning curves, and that the effect gradually diminishes as a subject adapts longer and the repulsive skewing of tuning curves counters the effect. Psychophysical data do not seem to support this prediction. The tilt aftereffect has been shown to increase monotonically with the duration of adaptation for up to an hour (Greenlee \& Magnussen, 1987). In addition, there is no reason to expect a quantitative agreement between the predicted aftereffect based on the cat data and the observed aftereffect in humans because of differences in species, stimulus parameters, and experimental paradigm. Despite these caveats, Jin et al.'s (2005) model provides an important framework for linking physiology and psychophysics that may prove useful in future studies.

As mentioned in Introduction, the V1 plasticity data are somewhat controversial. For example, Ghose et al. (2002) failed to find learning-induced changes of orientation tuning reported by Schoups et al. (2001), and we previously discussed possible causes 
of this discrepancy (Teich \& Qian, 2003a). However, as long as any of the six plasticity properties mentioned in Introduction exists in V1, our study supports the notion of intracortical sharpening of broadly tuned feedforward input. Tuning changes similar to those reported by Schoups et al. (2001) have also been found in V4 (Yang \& Maunsell, 2004; Raiguel et al., 2006). Thus, our study may also be relevant to orientation selectivity in higher cortical areas, suggesting intracortical sharpening as a mechanism for (partially) maintaining orientation tuning along the visual hierarchy.

In conclusion, we have systematically examined the plastic properties of the MFM and the MRM under plausible synaptic modification schemes. We find that the MRM can explain all the observed tuning curve changes induced by learning and adaptation, while the MFM cannot. Our work provides the first simple cell model for orientation plasticity and complements our previous simulations of plasticity in a complex cell model. Furthermore, our work reveals that the mere presence of recurrent cortical interactions is insufficient to explain orientation plasticity; rather, the recurrent interactions have to form a Mexican hat that sharpens broadly tuned feedforward input. Our work offers guidance on how to use plasticity to distinguish between different mechanisms of orientation selectivity. Future studies are needed to resolve this issue and to better link physiology and psychophysics of adaptation.

\section{Appendix 1}

In this appendix, we present some implementation details for the MFM and MRM.

In the MFM, the recipient V1 cell can be either excitatory ("e") or antiphase inhibitory ("ai"). There are a total of five weighting factors denoted by $w_{\mathrm{e} \rightarrow \mathrm{e}}, w_{\mathrm{e} \rightarrow \mathrm{ai}}, w_{\mathrm{ai} \rightarrow \mathrm{e}}, w_{\mathrm{f} \rightarrow \mathrm{e}}$, and $w_{\mathrm{f} \rightarrow \mathrm{ai}}$, which represent weights for excitatory to excitatory, excitatory to antiphase inhibitory, antiphase inhibitory to excitatory, feedforward to excitatory, and feedforward to antiphase inhibitory connections, respectively. Their values are identical to those in Teich and Qian (2006), with $w_{\mathrm{e} \rightarrow \mathrm{e}}=0.13, w_{\mathrm{e} \rightarrow \mathrm{ai}}=0.15, w_{\mathrm{ai} \rightarrow \mathrm{e}}=0.22, w_{\mathrm{f} \rightarrow \mathrm{e}}=0.1$, and $w_{\mathrm{f} \rightarrow \mathrm{ai}}=0.1$. Note that there are no inhibitory to inhibitory connections in the MFM (Troyer et al., 1998), so $w_{\mathrm{ai} \rightarrow \mathrm{ai}}=0$. The membrane potential $(V)$ of each cortical excitatory cell in the MFM is updated according to the differential equation (Carandini \& Ringach, 1997; Teich \& Qian, 2003a):

$$
\tau \partial V / \partial t+V=V_{\mathrm{f}}+V_{\mathrm{e}}-V_{\mathrm{ai}}
$$

where $\tau$ is the membrane time constant (15 $\mathrm{ms}$ in all simulations), and $V_{\mathrm{f}}, V_{\mathrm{e}}$, and $V_{\mathrm{ai}}$ are the synaptic potentials generated by the feedforward, cortical excitatory, and antiphase inhibitory input. For a given excitatory cell indexed at $(\theta, \phi)$, these potentials are given by:

$$
\begin{gathered}
V_{\mathrm{f}}=\sum_{x, y} w_{\mathrm{f} \rightarrow \mathrm{e}} \times C(x, y ; \theta, \phi) \times R_{\operatorname{lgn}}(x, y) \\
V_{\mathrm{e}}=\sum_{\theta^{\prime} \phi^{\prime}} w_{\mathrm{e} \rightarrow \mathrm{e}} \times C_{\mathrm{e} \rightarrow \mathrm{e}}\left(\theta^{\prime}, \phi^{\prime} ; \theta, \phi\right) \times R_{\mathrm{e}}\left(\theta^{\prime}, \phi^{\prime}\right) \\
V_{\mathrm{ai}}=\sum_{\theta^{\prime} \phi^{\prime}} w_{\mathrm{ai} \rightarrow \mathrm{e}} \times C_{\mathrm{ai} \rightarrow \mathrm{e}}\left(\theta^{\prime}, \phi^{\prime} ; \theta, \phi\right) \times R_{\mathrm{ai}}\left(\theta^{\prime}, \phi^{\prime}\right),
\end{gathered}
$$

where $R_{\operatorname{lgn}}(x, y)$ is the firing rate of the LGN cell at spatial position $(x, y)$, and $R_{\mathrm{e}}\left(\theta^{\prime}, \phi^{\prime}\right)$ and $R_{\mathrm{ai}}\left(\theta^{\prime}, \phi^{\prime}\right)$ represent the firing rate of an excitatory cell and an antiphase inhibitory cell indexed at $\left(\theta^{\prime}, \phi^{\prime}\right)$, respectively. The summations in the three equations cover LGN cells, cortical excitatory cells, and cortical antiphase inhibitory cells, respectively. All $C$ s represent normalized connectivity patterns; $C(x$, $y ; \theta, \phi)$ is determined according to the explanation after eqn. (2), and $C_{\mathrm{e} \rightarrow \mathrm{e}}\left(\theta^{\prime}, \phi^{\prime} ; \theta, \phi\right)$ and $C_{\mathrm{ai} \rightarrow \mathrm{e}}\left(\theta^{\prime}, \phi^{\prime} ; \theta, \phi\right)$ are set according to eqn. (4) in the Materials and methods section.

The membrane potential for each antiphase inhibitory V1 cell is calculated in the same way but with $w_{\mathrm{f} \rightarrow \mathrm{e}}, w_{\mathrm{e} \rightarrow \mathrm{e}}$, and $w_{\mathrm{ai} \rightarrow \mathrm{e}}$ replaced by $w_{\mathrm{f} \rightarrow \mathrm{ai}}, w_{\mathrm{e} \rightarrow \mathrm{ai}}$, and $w_{\mathrm{ai} \rightarrow \mathrm{ai}}(=0)$, respectively.

In the MRM, there are excitatory ("e"), recurrent inhibitory ("ri"), and antiphase inhibitory ("ai") cells and a total of 10 weighting factors denoted by $w_{\mathrm{e} \rightarrow \mathrm{e}}, w_{\mathrm{e} \rightarrow \mathrm{ri}}, w_{\mathrm{e} \rightarrow \mathrm{ai}}, w_{\mathrm{ri} \rightarrow \mathrm{e}}, w_{\mathrm{ri} \rightarrow \mathrm{ri}}$, $w_{\mathrm{ai} \rightarrow \mathrm{e}}, w_{\mathrm{ai} \rightarrow \mathrm{ri}}, w_{\mathrm{f} \rightarrow \mathrm{e}}, w_{\mathrm{f} \rightarrow \mathrm{ri}}$, and $w_{\mathrm{f} \rightarrow \mathrm{ai}}$. The specific values of these weights depend on whether we are running a "simple cell" or "complex cell" version of the MRM. For the simple cell MRM, $w_{\mathrm{e} \rightarrow \mathrm{e}}=3.2, w_{\mathrm{e} \rightarrow \mathrm{ri}}=3.2, w_{\mathrm{e} \rightarrow \mathrm{ai}}=0.7, w_{\mathrm{ri} \rightarrow \mathrm{e}}=3.5, w_{\mathrm{ri} \rightarrow \mathrm{ri}}=3.5$, $w_{\mathrm{ai} \rightarrow \mathrm{e}}=0.2, w_{\mathrm{ai} \rightarrow \mathrm{ri}}=0.2, w_{\mathrm{f} \rightarrow \mathrm{e}}=0.07, w_{\mathrm{f} \rightarrow \mathrm{ri}}=0.07$, and $w_{\mathrm{f} \rightarrow \mathrm{ai}}=$ 0.07 . The complex cell MRM is identical to the simple cell version, except that recurrent inhibition weights $\left(w_{\mathrm{ri} \rightarrow \mathrm{e}}\right.$ and $\left.w_{\mathrm{ri} \rightarrow \mathrm{ri}}\right)$ are increased and antiphase inhibition weights $\left(w_{\mathrm{ai} \rightarrow \mathrm{e}}\right.$ and $\left.w_{\mathrm{ai} \rightarrow \mathrm{ri}}\right)$ are decreased (see Materials and methods). All these values are identical to those in Teich and Qian (2006). The membrane potential ( $V$ ) of each cortical excitatory cell in the MRM is updated according to the differential equation (Carandini \& Ringach, 1997; Teich \& Qian, 2003a):

$$
\tau \partial V / \partial t+V=V_{\mathrm{f}}+V_{\mathrm{e}}-V_{\mathrm{ri}}-V_{\mathrm{ai}},
$$

where $V_{\mathrm{f}}$ and $V_{\text {ai }}$ are as defined above, and $V_{\mathrm{e}}$ and $V_{\mathrm{ri}}$ are the synaptic potential generated by the recurrent excitatory and inhibitory input. For a cortical excitatory cell indexed at $(\theta, \phi)$, the latter two potentials are given by

$$
\begin{gathered}
V_{\mathrm{e}}=\sum_{\theta^{\prime} \phi^{\prime}} w_{\mathrm{e} \rightarrow \mathrm{e}} \times C_{\mathrm{e} \rightarrow \mathrm{e}}\left(\theta^{\prime}, \phi^{\prime} ; \theta, \phi\right) \times R_{\mathrm{e}}\left(\theta^{\prime}, \phi^{\prime}\right) \\
V_{\mathrm{ri}}=\sum_{\theta^{\prime} \phi^{\prime}} w_{\mathrm{ri} \rightarrow \mathrm{e}} \times C_{\mathrm{ri} \rightarrow \mathrm{e}}\left(\theta^{\prime}, \phi^{\prime} ; \theta, \phi\right) \times R_{\mathrm{ri}}\left(\theta^{\prime}, \phi^{\prime}\right),
\end{gathered}
$$

where the summations cover the recurrent excitatory and inhibitory cells indexed at $\left(\theta^{\prime}, \phi^{\prime}\right)$, respectively; the normalized connectivity patterns $C_{\mathrm{e} \rightarrow \mathrm{e}}\left(\theta^{\prime}, \phi^{\prime} ; \theta, \phi\right)$ and $C_{\mathrm{ri} \rightarrow \mathrm{e}}\left(\theta^{\prime}, \phi^{\prime} ; \theta, \phi\right)$ are determined according to eqns. (5) and (6) from the Materials and methods section, respectively; and $R_{\mathrm{e}}\left(\theta^{\prime}, \phi^{\prime}\right)$ and $R_{\mathrm{ri}}\left(\theta^{\prime}, \phi^{\prime}\right)$ represent the firing rate of the recurrent excitatory and inhibitory cells, respectively.

The same equations are used to update the membrane potential of each recurrent inhibitory V1 cell but with $w_{\mathrm{e} \rightarrow \mathrm{e}}$ and $w_{\mathrm{ri} \rightarrow \mathrm{e}}$ replaced by $w_{\mathrm{e} \rightarrow \mathrm{ri}}$ and $w_{\mathrm{ri} \rightarrow \mathrm{ri}}$. The equations for updating each antiphase inhibitory V1 cell are the same as those in the MFM described above. Thus, as in the MFM, the "ai" cells in the MRM receive same-phase excitation from " $\mathrm{e}$ " cells and deliver antiphase inhibition to "e" cells. In addition, the "ai" cells in the MRM deliver antiphase inhibition to "ri" cells.

The weighting factors $(w s)$ in the above equations are constants for baseline simulations but depend on the preferred orientation of either pre- or postsynaptic cells $\left(\theta^{\prime}\right.$ or $\left.\theta\right)$ for plasticity simulations; see "Changing connection weights" in the Materials and methods section for a full explanation.

The interval $(\Delta t)$ at which we integrated all differential equations is $1 \mathrm{~ms}$ in all simulations. Our models directly relate membrane 
potential to firing rate via the threshold function with a gain factor $\alpha$ (Carandini \& Ringach, 1997; Teich \& Qian, 2003a):

$$
R=\alpha \max (V, 0)
$$

The $\alpha$ value is 5 for excitatory cells and 8 for antiphase inhibitory cells in our MFM and 6.5 for all cortical cells in our MRM. These values are chosen to produce the right baseline and maximum firing rates in each model but can be altered without changing the overall qualitative results.

\section{Appendix 2}

The opposite directions of skewing between tuning curves and population responses have been shown graphically (Gilbert \& Wiesel, 1990; Yao \& Dan, 2001). Here, we give a simple algebraic demonstration. Let $R(\theta, \varphi)$ represent the mean response of cells with preferred orientation $\theta$ to a stimulus orientation $\varphi$. For simplicity, we focus on skewing without considering activity reduction at the adapted orientation or other inhomogeneity across different orientations. Then, $R(\theta, \varphi)$ only depends on the difference between $\theta$ and $\varphi: R(\theta, \varphi)=R(\theta-\varphi)$. Now note that a tuning curve is $R(\theta-\varphi)$ as a function of $\varphi$ for a fixed $\theta$, while the population response is $R(\theta-$ $\varphi)$ as a function of $\theta$ for a fixed $\varphi$. Therefore, the tuning curve and populations response are related by $R(x)$ and $R(-x)$, respectively. In other words, they are mirror reflections of each other, with opposite directions of skewing. When different orientations are not represented homogenously as is the case of adaptation, the argument is only an approximation.

\section{Acknowledgment}

This work was supported by National Eye Institute grants R01 EY-016270 and T32 EY-13933.

\section{References}

Аввотт, L.F. \& Nelson, S.B. (2000). Synaptic plasticity: Taming the beast. Nature Neuroscience 3(Suppl.), 1178-1183.

Albrecht, D.G., Farrar, S.B. \& Hamilton, D.B. (1984). Spatial contrast adaptation characteristics of neurones recorded in the cat's visual cortex. The Journal of Physiology 347, 713-739.

Ben-Yishai, R., Bar-OR, R.L. \& Sompolinsky, H. (1995). Theory of orientation tuning in visual cortex. Proceedings of the National Academy of Sciences of the United States of America 92, 3844-3848.

Betz, W.J. (1970). Depression of transmitter release at the neuromuscular junction of the frog. The Journal of Physiology 206, 629-644.

BonDS, A.B. (1991). Temporal dynamics of contrast gain in single cells of the cat striate cortex. Visual Neuroscience 6, 239-255.

Carandini, M. \& Ferster, D. (1997). A tonic hyperpolarization underlying contrast adaptation in cat visual cortex. [see comment]. Science 276, 949-952.

Carandini, M. \& Ringach, D.L. (1997). Predictions of a recurrent model of orientation selectivity. Vision Research 37, 3061-3071.

Chen, Y., WANG, Y. \& Qian, N. (2001). Modeling V1 disparity tuning to time-varying stimuli. Journal of Neurophysiology 86, 143-155.

Chung, S. \& Ferster, D. (1998). Strength and orientation tuning of the thalamic input to simple cells revealed by electrically evoked cortical suppression. Neuron 20, 1177-1189.

Clifford, C.W., Wenderoth, P. \& Spehar, B. (2000). A functional angle on some after-effects in cortical vision. Proceedings of the Royal Society of London. Series B, Biological Sciences 267, 1705-1710.

DeAN, A.F. (1983). Adaptation-induced alteration of the relation between response amplitude and contrast in cat striate cortical neurones. Vision Research 23, 249-256.

Douglas, R.J., Кoch, C., Mahowald, M., Martin, K.A. \& Suarez, H.H. (1995). Recurrent excitation in neocortical circuits. Science 269, 981-985.
Dragoi, V., Rivadulla, C. \& SuR, M. (2001). Foci of orientation plasticity in visual cortex. Nature 411, 80-86.

Dragoi, V., Sharma, J., Miller, E.K. \& Sur, M. (2002). Dynamics of neuronal sensitivity in visual cortex and local feature discrimination. Nature Neuroscience 5, 883-891.

DragoI, V., Sharma, J. \& Sur, M. (2000). Adaptation-induced plasticity of orientation tuning in adult visual cortex. Neuron 28, 287-298.

Feldman, D.E. (2002). Synapses, scaling and homeostasis in vivo. [comment]. Nature Neuroscience 5, 712-714.

Felsen, G., Shen, Y.S., Yao, H., Spor, G., Li, C. \& Dan, Y. (2002). Dynamic modification of cortical orientation tuning mediated by recurrent connections. Neuron 36, 945-954.

Ferster, D., Chung, S. \& Wheat, H. (1996). Orientation selectivity of thalamic input to simple cells of cat visual cortex. [see comment]. Nature 380, 249-252.

Ferster, D. \& Miller, K.D. (2000). Neural mechanisms of orientation selectivity in the visual cortex. Annual Review of Neuroscience 23, 441-471.

Ghose, G.M., YANG, T. \& Maunsell, J.H. (2002). Physiological correlates of perceptual learning in monkey V1 and V2. Journal of Neurophysiology 87, 1867-1888.

Giaschi, D., Douglas, R., Marlin, S. \& Cynader, M. (1993). The time course of direction-selective adaptation in simple and complex cells in cat striate cortex. Journal of Neurophysiology 70, 2024-2034.

GiBSON, J.J. (1933). Adaptation, after-effect and contrast in the perception of curved lines. Journal of Experimental Psychology 16, 1-33.

GilberT, C.D. \& Wiesel, T.N. (1990). The influence of contextual stimuli on the orientation selectivity of cells in primary visual cortex of the cat. Vision Research 30, 1689-1701.

Greenlee, M.W. \& Magnussen, S. (1987). Saturation of the tilt aftereffect. Vision Research 27, 1041-1043.

Hammond, P., Mouat, G.S. \& Smith, A.T. (1988). Neural correlates of motion after-effects in cat striate cortical neurones: Monocular adaptation. Experimental Brain Research 72, 1-20.

HubeL, D.H. \& WiESEL, T.N. (1962). Receptive fields, binocular interaction and functional architecture in the cat's visual cortex. The Journal of Physiology 160, 106-154

Jin, D.Z., DragoI, V., Sur, M. \& Seung, H.S. (2005). Tilt aftereffect and adaptation-induced changes in orientation tuning in visual cortex. Journal of Neurophysiology 94, 4038-4050.

KoHn, A. \& MovshON, J.A. (2004). Adaptation changes the direction tuning of macaque MT neurons. Nature Neuroscience 7, 764-772.

Kusano, K. \& Landau, E.M. (1975). Depression and recovery of transmission at the squid giant synapse. The Journal of Physiology 245, 13-22.

Maffei, L., Fiorentini, A. \& Bisti, S. (1973). Neural correlate of perceptual adaptation to gratings. Science 182, 1036-1038.

Marlin, S.G., Hasan, S.J. \& Cynader, M.S. (1988). Direction-selective adaptation in simple and complex cells in cat striate cortex. Journal of Neurophysiology 59, 1314-1330.

Movshon, J.A. \& LenNiE, P. (1979). Pattern-selective adaptation in visual cortical neurones. Nature 278, 850-852.

Muller, J.R., Metha, A.B., Krauskopf, J. \& Lennie, P. (1999). Rapid adaptation in visual cortex to the structure of images. Science $\mathbf{2 8 5}, 1405$ 1408.

Ohzawa, I., Sclar, G. \& Freeman, R.D. (1982). Contrast gain control in the cat visual cortex. Nature 298, 266-268.

Ohzawa, I., Sclar, G. \& Freeman, R.D. (1985). Contrast gain control in the cat's visual system. Journal of Neurophysiology 54, 651-667.

Peters, A. \& Payne, B.R. (1993). Numerical relationships between geniculocortical afferents and pyramidal cell modules in cat primary visual cortex. Cerebral Cortex 3, 69-78.

Qian, N. \& Matthews, N. (1999). A physiological theory for visual perceptual learning of orientation discrimination. Society for Neuroscience Abstract 25, 1316.

Raiguel, S., Vogels, R., Mysore, S.G. \& Orban, G.A. (2006). Learning to see the difference specifically alters the most informative V4 neurons. [see comment]. Journal of Neuroscience 26, 6589-6602.

Regan, D. \& Beverley, K.I. (1985). Postadaptation orientation discrimination. Journal of the Optical Society of America. A, Optics, Image Science, and Vision 2, 147-155.

Schoups, A., Vogels, R., Qian, N. \& Orban, G. (2001). Practising orientation identification improves orientation coding in V1 neurons. Nature 412, 549-553.

Schoups, A.A., Vogels, R. \& Orban, G.A. (1998). Effects of perceptua learning in orientation discrimination on orientation coding in $\mathrm{v} 1$. Investigative Ophthalmology \& Visual Science 39, 684 
Sclar, G., Lennie, P. \& DePriest, D.D. (1989). Contrast adaptation in striate cortex of macaque. Vision Research 29, 747-755.

Somers, D.C., Nelson, S.B. \& Sur, M. (1995). An emergent model of orientation selectivity in cat visual cortical simple cells. Journal of Neuroscience 15, 5448-5465.

Sutherland, N.S. (1961). Figural after-effects and apparent size. Quarterly Journal of Experimental Psychology 13, 222-228.

TEICH, A.F. \& QIAN, N. (2003a). Learning and adaptation in a recurrent model of V1 orientation selectivity. Journal of Neurophysiology 89, 2086-2100.

TeICH, A.F. \& QIAN, N. (2003b). Modeling Learning- and AdaptationInduced Changes to V1 Orientation Tuning. Program No. 126.9. 2003 Abstract Viewer/Itinerary Planner. Washington, DC: Society for Neuroscience. Online.

TeICH, A.F. \& QIAN, N. (2006). Comparison among some models of orientation selectivity. Journal of Neurophysiology 96, 404-419.

TEICH, A.F. \& QIAN, N. (2007). Modeling Learning and Adaptation Induced Plasticity of Orientation Tuning in V1. Poster III-110. COSYNE 2007 Abstract Book.
Troyer, T.W., Krukowski, A.E., Priebe, N.J. \& Miller, K.D. (1998) Contrast-invariant orientation tuning in cat visual cortex: Thalamocortical input tuning and correlation-based intracortical connectivity. Journal of Neuroscience 18, 5908-5927.

Turrigiano, G.G. \& Nelson, S.B. (2000). Hebb and homeostasis in neuronal plasticity. Current Opinion in Neurobiology 10, 358-364.

Vidyasagar, T.R. \& Siguenza, J.A. (1985). Relationship between orientation tuning and spatial frequency in neurones of cat area 17. Experimental Brain Research 57, 628-631.

von der Heydt, R., Hanny, P. \& Adorjani, C. (1978). Movement aftereffects in the visual cortex. Archives Italiennes de Biologie 116, $248-254$.

Wolfe, J.M. (1984). Short test flashes produce large tilt aftereffects. Vision Research 24, 1959-1964.

YANG, T. \& MAunsell, J.H. (2004). The effect of perceptual learning on neuronal responses in monkey visual area V4. Journal of Neuroscience 24, 1617-1626.

YAO, H. \& DAN, Y. (2001). Stimulus timing-dependent plasticity in cortical processing of orientation. Neuron 32, 315-323. 